\title{
ON NONLOCAL PARABOLIC STEADY-STATE EQUATIONS OF COOPERATIVE OR COMPETING SYSTEMS
}

\author{
CHRISTOPH WALKER
}

\begin{abstract}
Some systems of parabolic equations with nonlocal initial conditions are studied. The systems arise when considering steady-state solutions to diffusive age-structured cooperative or competing species. Local and global bifurcation techniques are employed to derive a detailed description of the structure of positive coexistence solutions.
\end{abstract}

\section{InTRODUCTION AND MAIN RESUlts}

In this paper we characterize the structure of positive solutions to certain systems of coupled parabolic equations with nonlocal initial conditions. Such systems arise as steady-state equations of two agestructured diffusive populations which inhabit the same spatial region. The interaction between the two species is either of cooperative, competing, or predator-prey type leading to different structures of positive solutions. Denoting the density of the two species by $u=u(a, x) \geqslant 0$ and $v=v(a, x) \geqslant 0$ with $a \in\left(0, a_{m}\right)$ and $x \in \Omega \subset \mathbb{R}^{n}$ referring to age and spatial position, respectively, the models we shall focus on are of the form

$$
\begin{aligned}
& \partial_{a} u-\Delta_{D} u=-\alpha_{1} u^{2} \pm \alpha_{2} v u, \quad a \in\left(0, a_{m}\right), \quad x \in \Omega, \\
& \partial_{a} v-\Delta_{D} v=-\beta_{1} v^{2} \pm \beta_{2} u v, \quad a \in\left(0, a_{m}\right), \quad x \in \Omega,
\end{aligned}
$$

subject to the nonlocal initial conditions

$$
\begin{aligned}
& u(0, x)=\eta \int_{0}^{a_{m}} b_{1}(a) u(a, x) \mathrm{d} a, \quad x \in \Omega, \\
& v(0, x)=\xi \int_{0}^{a_{m}} b_{2}(a) v(a, x) \mathrm{d} a, \quad x \in \Omega .
\end{aligned}
$$

The equations are the steady-state equations of the corresponding time-dependent age-structured equations with diffusion. We refer to [30] for a recent survey on the formidable literature about age-structured population models.

The operator $-\Delta_{D}$ in (1.1), (1.2) stands for the negative Laplacian on $\Omega$ with subscript $D$ indicating that Dirichlet conditions

$$
u(a, x)=v(a, x)=0, \quad a \in\left(0, a_{m}\right), \quad x \in \partial \Omega,
$$

are imposed on the smooth boundary $\partial \Omega$ of the bounded domain $\Omega$. Normalization to 1 of the diffusion coefficients in (1.1), (1.2) is a purely notational simplification. The number $a_{m}>0$ denotes the maximal age of the species. Equations (1.3), (1.4) represent the age-boundary conditions reflecting that individuals with age zero are those created when a mother individual of any age $a \in\left(0, a_{m}\right)$ gives birth with rates $\eta b_{1}(a)$ or $\xi b_{2}(a)$. The functions $b_{j}=b_{j}(a) \geqslant 0$ describe the profiles of the fertility rates while the parameters $\eta, \xi>0$ measure their intensity without affecting the structure of the birth profiles. For easier statements of the results we assume the birth profiles

$$
b_{j} \in L_{\infty}^{+}\left(\left(0, a_{m}\right)\right) \text { with } b_{j}(a)>0 \text { for } a \text { near } a_{m}, \quad j=1,2,
$$

Key words and phrases. Bifurcation, diffusion, age structure, maximal regularity, Krein-Rutman. 
are normalized such that

$$
\int_{0}^{a_{m}} b_{j}(a) e^{-\lambda_{1} a} \mathrm{~d} a=1, \quad j=1,2,
$$

where $\lambda_{1}>0$ denotes the principal eigenvalue of $-\Delta_{D}$ on $\Omega$.

Assuming $\alpha_{1}, \alpha_{2}, \beta_{1}, \beta_{2}>0$, the form of the interaction between the two species is determined by the signs on the right hand side of equations (1.1), 1.2). Replacing \pm by a positive sign + in both of the equations (1.1) and (1.2) ) corresponds to a system (see (1.11), (1.12) below) where the two species are cooperative, while the case with \pm replaced by negative signs - in each equation (1.1) and (1.2) (see (1.14), (1.15) below) reflects a competition of the species. The case with mixed signs, e.g. a negative sign - in (1.1) instead of \pm and a positive sign + in (1.2) describes a predator-prey-system (see (1.17), (1.18) below) with a prey density $u$ and a predator density $v$.

This last case of a predator-prey-system was studied in [29] and local and global bifurcation phenomena of positive nontrivial solutions with respect to the parameters $\eta$ and $\xi$ were obtained. In the present paper, we shall derive global bifurcation results for the cooperative and the competition case. Depending on $\eta$ and $\xi$ we shall give a rather complete description of positive coexistence solutions, that is, of solutions $(u, v)$ with both components $u$ and $v$ positive and nontrivial. Moreover, we shall also improve the local bifurcation result [29, Thm.2.4] to a global one.

We like to point out that variants of the elliptic counterparts to (1.1)-(1.2) when age-structure is neglected from the outset have been intensively studied in the past, e.g. see [4, 5, 7, 8, 9, 11, 12, 13, 18, 19, 20, 21, 22, 24, 31]. Concerning equations for a single specie, e.g. variants of (1.1) subject to (1.3), we refer to [15, 16, 25, 26, 27, 28].

To state our results for the present situation, we shall keep $\xi$ fixed and regard $\eta$ as bifurcation parameter in the following. We thus write $(\eta, u, v)$ for solutions to (1.1)-(1.4) with $u, v$ belonging to the positive cone $\mathbb{W}_{q}^{+}$of

$$
\mathbb{W}_{q}:=L_{q}\left(\left(0, a_{m}\right), W_{q, D}^{2}(\Omega)\right) \cap W_{q}^{1}\left(\left(0, a_{m}\right), L_{q}(\Omega)\right)
$$

for $q>n+2$ fixed, but remark that all our solutions will have smooth components $u$, $v$ with respect to both $a \in J$ and $x \in \Omega$. We say that a continuum $\mathfrak{C}$ (i.e. a closed and connected set) in $\mathbb{R}^{+} \times \mathbb{W}_{q}^{+} \times \mathbb{W}_{q}^{+}$of solutions $(\eta, u, v)$ to (1.1)-(1.4) is unbounded with respect to $\eta$, provided the $\eta$-projection of $\mathfrak{C}$ contains an interval of the form $\left(\eta_{0}, \infty\right)$ with $\eta_{0} \in \mathbb{R}^{+}$, and we say that $\mathfrak{C}$ is unbounded with respect to the u-component in $\mathbb{W}_{q}$ provided there is a sequence $\left(\left(\eta_{j}, u_{j}, v_{j}\right)\right)_{j \in \mathbb{N}}$ in $\mathfrak{C}$ with $\left\|u_{j}\right\|_{\mathbb{W}_{q}} \rightarrow \infty$ as $j \rightarrow \infty$. An analogous terminology shall be used if $\mathfrak{C}$ is unbounded with respect to the $v$-component.

Clearly, problem (1.1)-(1.4) decouples when taking either $u$ or $v$ to be zero. Noticing that Theorem A.4 from the appendix provides for each $\eta>1$ a unique solution $u_{\eta} \in \mathbb{W}_{q}^{+} \backslash\{0\}$ to

$$
\partial_{a} u-\Delta_{D} u=-\alpha_{1} u^{2}, \quad u(0, \cdot)=\eta \int_{0}^{a_{m}} b_{1}(a) u(a, \cdot) \mathrm{d} a,
$$

and similarly for each $\xi>1$ a unique solution $v_{\xi} \in \mathbb{W}_{q}^{+} \backslash\{0\}$ to

$$
\partial_{a} v-\Delta_{D} v=-\beta_{1} v^{2}, \quad v(0, \cdot)=\xi \int_{0}^{a_{m}} b_{2}(a) v(a, \cdot) \mathrm{d} a,
$$

there is, independent of what the signs \pm in (1.1), (1.2) are, for any $\xi \geqslant 0$ the trivial branch

$$
\mathfrak{B}_{0}:=\{(\eta, 0,0) ; \eta \geqslant 0\}
$$

and the semi-trivial branch

$$
\mathfrak{B}_{1}:=\left\{\left(\eta, u_{\eta}, 0\right) ; \eta>1\right\} \subset \mathbb{R}^{+} \times\left(\mathbb{W}_{q}^{+} \backslash\{0\}\right) \times \mathbb{W}_{q}^{+}
$$

of solutions. For $\xi>1$, an additional semi-trivial branch

$$
\mathfrak{B}_{2}:=\left\{\left(\eta, 0, v_{\xi}\right) ; \eta \geqslant 0\right\} \subset \mathbb{R}^{+} \times \mathbb{W}_{q}^{+} \times\left(\mathbb{W}_{q}^{+} \backslash\{0\}\right)
$$


exists. Depending on the signs \pm in (1.1), (1.2) we shall establish further local and global bifurcation of coexistence solutions from these semi-trivial branches.

1.1. Cooperative Systems. We first consider the cooperative case

$$
\begin{array}{lll}
\partial_{a} u-\Delta_{D} u=-\alpha_{1} u^{2}+\alpha_{2} v u, & a \in\left(0, a_{m}\right), & x \in \Omega, \\
\partial_{a} v-\Delta_{D} v=-\beta_{1} v^{2}+\beta_{2} u v, & a \in\left(0, a_{m}\right), & x \in \Omega,
\end{array}
$$

subject to the nonlocal initial conditions

$$
u(0, x)=\eta \int_{0}^{a_{m}} b_{1}(a) u(a, x) \mathrm{d} a, \quad v(0, x)=\xi \int_{0}^{a_{m}} b_{2}(a) v(a, x) \mathrm{d} a, \quad x \in \Omega .
$$

Recall that $\mathfrak{B}_{1}$ is the only semi-trivial branch of solutions to 1.11 - $(1.12)$ if $\xi<1$. Thus, if $\xi<1$ we shall derive bifurcation with respect to the parameter $\eta$ from the semi-trivial branch $\mathfrak{B}_{1}$ consisting of solutions of the form $\left(\eta, u_{\eta}, 0\right)$. The case $\xi<1$ is therefore more involved than the case $\xi>1$ where we shall establish a bifurcation from the semi-trivial branch $\mathfrak{B}_{2}$ consisting of solutions of the form $\left(\eta, 0, v_{\xi}\right)$.

Theorem 1.1. There is $\nu \in[0,1)$ with the property that for each $\xi \in(\nu, 1)$ there exists $\eta_{0}:=\eta_{0}(\xi)>1$ such that $\left(\eta_{0}, u_{\eta_{0}}, 0\right) \in \mathfrak{B}_{1}$ is a bifurcation point. There is an unbounded continuum $\mathfrak{C}_{1}$ of coexistence solutions $(\eta, u, v)$ in $\mathbb{R}^{+} \times\left(\mathbb{W}_{q}^{+} \backslash\{0\}\right) \times\left(\mathbb{W}_{q}^{+} \backslash\{0\}\right)$ to (1.11)-(1.12) subject to (1.3)-(1.4) emanating from $\left(\eta_{0}, u_{\eta_{0}}, 0\right)$. Near the branch $\mathfrak{B}_{1}$, the continuum $\mathfrak{C}_{1}$ is a continuous curve. There is no other bifurcation point on $\mathfrak{B}_{1}$ to positive coexistence solutions.

The precise values of $\nu$ and of $\eta_{0}(\xi)>1$ are related to spectral radii of some operators and are given in (3.2) and (3.3), respectively. Actually, we conjecture that $\nu=0$, see Remark 3.1 .

If $\xi>1$, then a global continuum of coexistence solutions emanates from the branch $\mathfrak{B}_{2}$ :

Theorem 1.2. Given $\xi>1$, there is $\eta_{1}:=\eta_{1}(\xi) \in(0,1)$ such that $\left(\eta_{1}, 0, v_{\xi}\right) \in \mathfrak{B}_{2}$ is a bifurcation point. An unbounded continuum $\mathfrak{C}_{2}$ of coexistence solutions $(\eta, u, v)$ in $\mathbb{R}^{+} \times\left(\mathbb{W}_{q}^{+} \backslash\{0\}\right) \times\left(\mathbb{W}_{q}^{+} \backslash\{0\}\right)$ to (1.11)-(1.12) subject to (1.3)-(1.4) emanates from $\left(\eta_{1}, 0, v_{\xi}\right)$. Near the branch $\mathfrak{B}_{2}$, the continuum $\mathfrak{C}_{2}$ is a continuous curve. There is no other bifurcation point on $\mathfrak{B}_{2}$ or on $\mathfrak{B}_{1}$ to positive coexistence solutions.

The precise value of $\eta_{1}(\xi)$ is given in (4.1). We can give a more specific characterization of the global nature of the the continua $\mathfrak{C}_{1}$ and $\mathfrak{C}_{2}$ :

Corollary 1.3. The global continua $\mathfrak{C}_{1}$ and $\mathfrak{C}_{2}$ provided by Theorem 1.1 and Theorem 1.2 respectively, are unbounded with respect to both the parameter $\eta$ and the u-component in $\mathbb{W}_{q}$, or with respect to the $v$-component in $\mathbb{W}_{q}$. If

$$
b_{2} \in L_{1}\left(\left(0, a_{m}\right),\left(1-e^{-s a}\right)^{-1} \mathrm{~d} a\right)
$$

for some $s>0$, then they are unbounded with respect to the u-component in $\mathbb{W}_{q}$.

1.2. Competing Systems. Next we consider the case of two competing species:

$$
\begin{array}{lll}
\partial_{a} u-\Delta_{D} u=-\alpha_{1} u^{2}-\alpha_{2} v u, & a \in\left(0, a_{m}\right), & x \in \Omega, \\
\partial_{a} v-\Delta_{D} v=-\beta_{1} v^{2}-\beta_{2} u v, & a \in\left(0, a_{m}\right), & x \in \Omega,
\end{array}
$$

subject to the initial conditions

$$
u(0, x)=\eta \int_{0}^{a_{m}} b_{1}(a) u(a, x) \mathrm{d} a, \quad v(0, x)=\xi \int_{0}^{a_{m}} b_{2}(a) v(a, x) \mathrm{d} a, \quad x \in \Omega .
$$

The following theorem characterizes the competition coexistence solutions.

Theorem 1.4. If $\xi \leqslant 1$, then there is no coexistence solution $(\eta, u, v) \in \mathbb{R}^{+} \times\left(\mathbb{W}_{q}^{+} \backslash\{0\}\right) \times\left(\mathbb{W}_{q}^{+} \backslash\{0\}\right)$ to (1.14)-(1.15) subject to (1.3)-(1.4). If $\xi>1$, then there is $\eta_{2}:=\eta_{2}(\xi)>1$ such that $\left(\eta_{2}, 0, v_{\xi}\right) \in \mathfrak{B}_{2}$ is a bifurcation point. A continuum $\mathfrak{C}_{3}$ of positive coexistence solutions in $\mathbb{R}^{+} \times\left(\mathbb{W}_{q}^{+} \backslash\{0\}\right) \times\left(\mathbb{W}_{q}^{+} \backslash\{0\}\right)$ emanates from $\left(\eta_{2}, 0, v_{\xi}\right)$ satisfying the alternative 
(a) $\mathfrak{C}_{3}$ joins $\mathfrak{B}_{2}$ with $\mathfrak{B}_{1}$, or

(b) $\mathfrak{C}_{3}$ is unbounded with respect to the parameter $\eta$,

and near the bifurcation point $\left(\eta_{2}, 0, v_{\xi}\right)$, the continuum $\mathfrak{C}_{3}$ is a continuous curve. There exists some $N>1$ such that alternative (a) occurs for each $\xi \in(1, N)$. Moreover, if

$$
\beta_{2} \geqslant \alpha_{1}, \quad \beta_{1} \geqslant \alpha_{2}, \quad b_{1} \geqslant b_{2} \text { on }\left(0, a_{m}\right),
$$

then the $\eta$-projection of $\mathfrak{C}_{3}$ is contained in the interval $(1, \xi]$; in particular, alternative (a) occurs for each $\xi>1$.

The value of $\eta_{2}(\xi)$ as well as the value of $\eta_{3}:=\eta_{3}(\xi)$ corresponding to the point $\left(\eta_{3}, u_{\eta_{3}}, 0\right) \in \mathfrak{B}_{1}$ where $\mathfrak{C}_{3}$ joins up with $\mathfrak{B}_{1}$ if alternative (a) occurs, are determined exactly, see (5.2) and (5.6). Actually, we conjecture $N=\infty$ even if (1.16) does not hold, see Remark 5.5 Observe that 1.16 implies a biological advantage of the specie with density $u$ due to a higher birth but lower death rate.

1.3. Predator-Prey-Systems. The case of a predator-prey-system,

$$
\begin{array}{lll}
\partial_{a} u-\Delta_{D} u=-\alpha_{1} u^{2}-\alpha_{2} v u, & a \in\left(0, a_{m}\right), & x \in \Omega, \\
\partial_{a} v-\Delta_{D} v=-\beta_{1} v^{2}+\beta_{2} u v, & a \in\left(0, a_{m}\right), & x \in \Omega,
\end{array}
$$

subject to the initial conditions

$$
u(0, x)=\eta \int_{0}^{a_{m}} b_{1}(a) u(a, x) \mathrm{d} a, \quad v(0, x)=\xi \int_{0}^{a_{m}} b_{2}(a) v(a, x) \mathrm{d} a, \quad x \in \Omega,
$$

was studied in detail in [29]. A quite complete description of the structure of positive solutions was provided when $\xi$ is regarded as bifurcation parameter and $\eta>0$ is kept fixed [29, Thm.2.2] or when $\eta$ is regarded as bifurcation parameter and $\xi>1$ is kept fixed [Thm.2.3]. However, for the case $\xi<1$ being fixed with parameter $\eta$, a local bifurcation and thus a merely partial result was obtained in [29, Thm.2.4]. More precisely, provided that $\xi \in(\delta, 1)$ for some suitable $\delta \in[0,1)$, it was shown in [29, Thm.2.4] that there are $\varepsilon_{0}>0$ and a unique point $\left(\eta_{4}, u_{\eta_{4}}, 0\right)$ with $\eta_{4}:=\eta_{4}(\xi)>1$ on the semi-trivial branch $\mathfrak{B}_{1}$ such that a local continuous curve

$$
\mathfrak{C}_{4}:=\left\{(\eta(\varepsilon), u(\varepsilon), v(\varepsilon)) ; 0<\varepsilon<\varepsilon_{0}\right\} \subset \mathbb{R}^{+} \times\left(\mathbb{W}_{q}^{+} \backslash\{0\}\right) \times\left(\mathbb{W}_{q}^{+} \backslash\{0\}\right)
$$

of positive coexistence solutions bifurcates to the right from $\left(\eta_{4}, u_{\eta_{4}}, 0\right)$. Actually, this result can be improved:

Theorem 1.5. The branch $\mathfrak{C}_{4}$ extends to an unbounded continuum in $\mathbb{R}^{+} \times\left(\mathbb{W}_{q}^{+} \backslash\{0\}\right) \times\left(\mathbb{W}_{q}^{+} \backslash\{0\}\right)$ of positive coexistence solutions to (1.17)-(1.18) subject to (1.3)-(1.4). If (1.13) holds, then $\mathfrak{C}_{4}$ is unbounded with respect to the parameter $\eta$.

The proof of this theorem is along the lines of the one of Theorem 1.1 with only minor modifications necessary. We shall thus omit it here.

The outline of the present paper is as follows: In the next section, Section 2 , notations and some preliminary results are introduced. In Section 3 a detailed proof of Theorem 1.1 is provided so that the proof of Theorem 1.2 in Section 4 is basically a straightforward modification thereof and can thus be kept short. Section 5 is dedicated to the proof of Theorem 1.4 Appendix A contains some results regarding the semi-trivial branches induced by (1.7), (1.8) which are of importance for the proofs in Sections 3

\section{Notations ANd PRELIMINARIES}

Throughout we assume that $\Omega$ is a bounded and smooth domain in $\mathbb{R}^{n}$. We fix $q>n+2$ and set, for $\kappa>1 / q$,

$$
W_{q, D}^{\kappa}:=W_{q, D}^{\kappa}(\Omega):=\left\{u \in W_{q}^{\kappa} ; u=0 \text { on } \partial \Omega\right\}
$$


where $W_{q}^{\kappa}:=W_{q}^{\kappa}(\Omega)$ stands for the usual Sobolev-Slobodeckii spaces (for arbitrary $\kappa>0$ ) and values on the boundary are interpreted in the sense of traces. Recall from [2, III.Thm.4.10.2] and the Sobolev embedding theorem that

$$
\mathbb{W}_{q} \hookrightarrow C\left(\left[0, a_{m}\right], W_{q, D}^{2-2 / q}\right) \hookrightarrow C\left(\left[0, a_{m}\right], C^{1}(\bar{\Omega})\right)
$$

so that the trace $\gamma_{0} u:=u(0) \in W_{q, D}^{2-2 / q} \hookrightarrow C^{1}(\bar{\Omega})$ is well-defined for $u \in \mathbb{W}_{q}$. Moreover, since also

$$
\mathbb{W}_{q} \hookrightarrow W_{q}^{1}\left(\left(0, a_{m}\right), L_{q}\right) \hookrightarrow C^{1-1 / q}\left(\left[0, a_{m}\right], L_{q}\right)
$$

with $L_{q}:=L_{q}(\Omega)$, the interpolation inequality in [2] I.Thm.2.11.1] yields in fact

$$
\mathbb{W}_{q} \hookrightarrow C^{1-1 / q-\vartheta}\left(\left[0, a_{m}\right], W_{q}^{2 \vartheta}\right), \quad 0 \leqslant \vartheta \leqslant 1-1 / q
$$

Note that the interior, $\operatorname{int}\left(W_{q, D}^{2-2 / q,+}\right)$, of the positive cone $W_{q, D}^{2-2 / q,+}$ of $W_{q, D}^{2-2 / q}$ is not empty. We set

$$
\mathbb{L}_{q}:=L_{q}\left(\left(0, a_{m}\right), L_{q}(\Omega)\right) \text { and } \quad \dot{\mathbb{W}}_{q}^{+}:=\mathbb{W}_{q}^{+} \backslash\{0\} .
$$

Put $J:=\left[0, a_{m}\right]$. Given $\varrho>0$ and $h \in C^{\varrho}(J, C(\bar{\Omega}))$, we let $\Pi_{[h]}(a, \sigma), 0 \leqslant \sigma \leqslant a \leqslant a_{m}$, denote the unique parabolic evolution operator corresponding to $-\Delta_{D}+h \in C^{\varrho}\left(J, \mathcal{L}\left(W_{q, D}^{2}, L_{q}\right)\right)$, that is,

$$
z(a)=\Pi_{[h]}(a, \sigma) \Phi, \quad a \in\left(\sigma, a_{m}\right)
$$

defines the unique strong solution to

$$
\partial_{a} z-\Delta_{D} z+h z=0, \quad a \in\left(\sigma, a_{m}\right), \quad z(\sigma)=\Phi,
$$

for any given $\sigma \in\left(0, a_{m}\right)$ and $\Phi \in L_{q}$ (see [2, II.Cor.4.4.1]). Note that the evolution operator is positive, i.e.

$$
\Pi_{[h]}(a, \sigma) \Phi \in L_{q}^{+}, \quad 0 \leqslant \sigma \leqslant a \leqslant a_{m}, \quad \Phi \in L_{q}^{+} .
$$

As $J$ is a compact interval and $-\Delta_{D}$ has bounded imaginary powers, it follows from (2.1) and [2] (in particular, see I.Cor.1.3.2, III.Thm.4.8.7, III.Thm.4.10.10 therein) that the operator $-\Delta_{D}+h$ has maximal $L_{q}$-regularity, i.e. the operator

$$
\left(\partial_{a}-\Delta_{D}+h, \gamma_{0}\right) \in \mathcal{L}\left(\mathbb{W}_{q}, \mathbb{L}_{q} \times W_{q, D}^{2-2 / q}\right)
$$

is a toplinear isomorphism. In particular, $\Pi_{[h]}(\cdot, 0) \Phi \in \mathbb{W}_{q}$ for $\Phi \in W_{q, D}^{2-2 / q}$. We set

$$
H_{[h]}:=\int_{0}^{a_{m}} b_{1}(a) \Pi_{[h]}(a, 0) \mathrm{d} a, \quad \hat{H}_{[h]}:=\int_{0}^{a_{m}} b_{2}(a) \Pi_{[h]}(a, 0) \mathrm{d} a .
$$

Then $H_{[h]}$ and $\hat{H}_{[h]}$ belong to $\mathcal{K}\left(W_{q, D}^{2-2 / q}\right)$, that is, they define compact linear operators on $W_{q, D}^{2-2 / q}$, and they are strongly positive, that is, e.g.

$$
H_{[h]} \Phi \in \operatorname{int}\left(W_{q, D}^{2-2 / q,+}\right), \quad \Phi \in W_{q, D}^{2-2 / q,+} \backslash\{0\} .
$$

The corresponding spectral radii $r\left(H_{[h]}\right)$ and $r\left(\hat{H}_{[h]}\right)$ can thus be characterized according to the KreinRutman theorem [1, Thm.3.2] (see Lemma A.3 from the appendix). In particular, the normalizations (1.6) readily imply

$$
r\left(H_{[0]}\right)=r\left(\hat{H}_{[0]}\right)=1
$$

since any positive eigenfunction of $-\Delta_{D}$ is an eigenfunction of $H_{[h]}$ and $\hat{H}_{[h]}$ as well. It is worthwhile to point out that (2.2) warrants an equivalent formulation of a solution $(u, v) \in \mathbb{W}_{q} \times \mathbb{W}_{q}$ to (1.1)-(1.4) as

$$
\begin{array}{lll}
u(a)=\Pi_{\left[\alpha_{1} u \mp \alpha_{2} v\right]}(a, 0) u(0), & a \in J, & u(0)=\eta H_{\left[\alpha_{1} u \mp \alpha_{2} v\right]} u(0), \\
v(a)=\Pi_{\left[\beta_{1} v \mp \beta_{2} u\right]}(a, 0) v(0), & a \in J, & v(0)=\xi \hat{H}_{\left[\beta_{1} v \mp \beta_{2} u\right]} v(0) .
\end{array}
$$


Observe that $u, v$ are nonzero or nonnegative provided $u(0), v(0)$ are nonzero or nonnegative. Hence, if $(u, v) \in \mathbb{W}_{q}^{+} \times \mathbb{W}_{q}^{+}$solves (1.1)-(1.4), then

$$
\begin{aligned}
& \eta r\left(H_{\left[\alpha_{1} u \mp \alpha_{2} v\right]}\right)=1 \quad \text { if } \quad u(0) \in W_{q, D}^{2-2 / q,+} \backslash\{0\}, \\
& \xi r\left(\hat{H}_{\left[\beta_{1} v \mp \beta_{2} u\right]}\right)=1 \quad \text { if } \quad v(0) \in W_{q, D}^{2-2 / q,+} \backslash\{0\},
\end{aligned}
$$

owing to Lemma A.3. In particular, we have

$$
\eta r\left(H_{\left[\alpha_{1} u_{\eta}\right]}\right)=\xi r\left(\hat{H}_{\left[\beta_{1} v_{\xi}\right]}\right)=1, \quad \eta, \xi>1
$$

by (1.7) and (1.8) since $u_{\eta}(0), v_{\xi}(0) \in W_{q, D}^{2-2 / q,+} \backslash\{0\}$. We conclude this section with the following auxiliary result:

Lemma 2.1. Given $M>0$ there is $c(M)>0$ such that

$$
\|u(a)\|_{\infty}+\|v(a)\|_{\infty} \leqslant M, \quad a \in J
$$

implies

$$
\|u\|_{\mathbb{W}_{q}} \leqslant c(M)(\eta+1), \quad\|v\|_{\mathbb{W}_{q}} \leqslant c(M)(\xi+1)
$$

for any solution $(u, v) \in \mathbb{W}_{q}^{+} \times \mathbb{W}_{q}^{+}$to (1.1)-(1.4) with $\eta, \xi>0$.

Proof. If $(u, v) \in \mathbb{W}_{q}^{+} \times \mathbb{W}_{q}^{+}$solves (1.1)-(1.4), we derive from (1.1), (2.10), and the property of maximal $L_{q}$-regularity of $-\Delta_{D}$ that there is $c_{0}(M)>0$ such that

$$
\|u\|_{\mathbb{W}_{q}} \leqslant c\left(\|u(0)\|_{W_{q, D}^{2-2 / q}}+\left\|-\alpha_{1} u^{2} \pm \alpha_{2} u v\right\|_{\mathbb{L}_{q}}\right) \leqslant c_{0}(M)\left(\|u(0)\|_{W_{q, D}^{2-2 / q}}+1\right) .
$$

Writing (1.1) in the form

$$
u(a)=e^{a \Delta_{D}} u(0)+\int_{0}^{a} e^{(a-\sigma) \Delta_{D}}\left(-\alpha_{1} u(\sigma)^{2} \pm \alpha_{2} u(\sigma) v(\sigma)\right) \mathrm{d} \sigma, \quad a \in J,
$$

and using $\left\|e^{a \Delta_{D}}\right\|_{\mathcal{L}\left(L_{q}, W_{q, D}^{2-2 / q}\right)} \leqslant c a^{1 / q-1}$ for $a>0$, we obtain from (1.3) and (2.10)

$$
\begin{aligned}
\|u(0)\|_{W_{q, D}^{2-2 / q}} \leqslant & \eta\left\|b_{1}\right\|_{\infty} \int_{0}^{a_{m}}\left\|e^{a \Delta_{D}}\right\|_{\mathcal{L}\left(L_{q}, W_{q, D}^{2-2 / q}\right)}\|u(0)\|_{L_{q}} \mathrm{~d} a \\
& +\eta\left\|b_{1}\right\|_{\infty} \int_{0}^{a_{m}} \int_{0}^{a}\left\|e^{(a-\sigma) \Delta_{D}}\right\|_{\mathcal{L}\left(L_{q}, W_{q, D}^{2-2 / q}\right)}\left(\left\|\alpha_{1} u(\sigma)^{2}\right\|_{L_{q}}+\left\|\alpha_{2} u(\sigma) v(\sigma)\right\|_{L_{q}}\right) \mathrm{d} \sigma \mathrm{d} a \\
\leqslant & c_{1}(M) \eta .
\end{aligned}
$$

Consequently, $\|u\|_{\mathbb{W}_{q}} \leqslant c(M)(\eta+1)$. Similarly we deduce $\|v\|_{\mathbb{W}_{q}} \leqslant c(M)(\xi+1)$.

\section{Cooperative Systems with $\xi<1$ : Proof of Theorem 1.1}

We focus our attention on (1.11)-(1.12) subject to (1.3)-(1.4) when $\xi<1$. First we show local bifurcation of a continuous curve from $\mathfrak{B}_{1}$ by using the results of Crandall-Rabinowitz [10]. We remark that

$$
\left(\eta \mapsto r\left(\hat{H}_{\left[-\beta_{2} u_{\eta}\right]}\right)\right) \in C((1, \infty),(1, \infty)) \text { is strictly increasing }, \quad \lim _{\eta \rightarrow 1} r\left(\hat{H}_{\left[-\beta_{2} u_{\eta}\right]}\right)=1
$$

according to Lemma A.3, Theorem A.4, and (2.4), so

$$
\nu:=\frac{1}{\lim _{\eta \rightarrow \infty} r\left(\hat{H}_{\left[-\beta_{2} u_{\eta}\right]}\right)} \in[0,1)
$$

is well-defined.

Remark 3.1. As $\left\|u_{\eta}\right\|_{\infty} \rightarrow \infty$ for $\eta \rightarrow \infty$ by Theorem A.4 we conjecture $\nu=0$ in (3.2). 
For the remainder of this section we fix $\xi \in(\nu, 1)$. The observations above ensure the existence of a unique value $\eta_{0}:=\eta_{0}(\xi)>1$ for which

$$
\xi r\left(\hat{H}_{\left[-\beta_{2} u_{\eta_{0}}\right]}\right)=1
$$

Note then that

$$
\operatorname{ker}\left(1-\xi \hat{H}_{\left[-\beta_{2} u_{\eta_{0}}\right]}\right)=\operatorname{span}\left\{\Psi_{0}\right\} \quad \text { with } \quad \Psi_{0} \in \operatorname{int}\left(W_{q, D}^{2-2 / q,+}\right)
$$

by the Krein-Rutman theorem. With these notations we have:

Lemma 3.2. There is a local continuous curve $\mathfrak{C}_{1} \subset \mathbb{R}^{+} \times \dot{\mathbb{W}}_{q}^{+} \times \dot{\mathbb{W}}_{q}^{+}$of coexistence solutions to (1.11)(1.12) subject to (1.3)-(1.4) bifurcating from $\left(\eta_{0}, u_{\eta_{0}}, 0\right) \in \mathfrak{B}_{1}$, and all positive coexistence solutions near $\left(\eta_{0}, u_{\eta_{0}}, 0\right)$ lie on this curve.

Proof. The proof is in the spirit of the one of [29, Prop.2.7]. We are linearizing (1.11)-(1.12) around $\left(\eta_{0}, u_{\eta_{0}}, 0\right) \in \mathfrak{B}_{1}$. For this observe that $(\eta, u, v)=\left(\eta, u_{\eta}+w, v\right) \in \mathbb{R} \times \mathbb{W}_{q} \times \mathbb{W}_{q}$ solves (1.11)-(1.12) subject to (1.3)-(1.4) if and only if $(\eta, w, v) \in \mathbb{R} \times \mathbb{W}_{q} \times \mathbb{W}_{q}$ solves

$$
\begin{array}{lr}
\partial_{a} w-\Delta_{D} w=-\alpha_{1} w^{2}-2 \alpha_{1} u_{\eta} w+\alpha_{2} v\left(u_{\eta}+w\right), & w(0)=\eta W, \\
\partial_{a} v-\Delta_{D} v=-\beta_{1} v^{2}+\beta_{2} v\left(u_{\eta}+w\right), & v(0)=\xi V,
\end{array}
$$

where we slightly abuse notation by writing

$$
W:=\int_{0}^{a_{m}} b_{1}(a) w(a) \mathrm{d} a, \quad V:=\int_{0}^{a_{m}} b_{2}(a) v(a) \mathrm{d} a
$$

when $w, v \in \mathbb{W}_{q}$. We shall use this notation also for other capital letters since it will always be clear from the context, which of the profiles $b_{1}$ or $b_{2}$ is meant. Using maximal $L_{q}$-regularity of $-\Delta_{D}$, we may introduce the operator

$$
T:=\left(\partial_{a}-\Delta_{D}, \gamma_{0}\right)^{-1} \in \mathcal{L}\left(\mathbb{L}_{q} \times W_{q, D}^{2-2 / q}, \mathbb{W}_{q}\right)
$$

so that the solutions to (3.5)-(3.6) are the zeros of the function

$$
F(\eta, w, u):=\left(\begin{array}{c}
w-T\left(-\alpha_{1} w^{2}-2 \alpha_{1} u_{\eta} w+\alpha_{2} v\left(u_{\eta}+w\right), \eta W\right) \\
v-T\left(-\beta_{1} v^{2}+\beta_{2} v\left(u_{\eta}+w\right), \xi V\right)
\end{array}\right) .
$$

Observe that

$$
F \in C^{2}\left((1, \infty) \times \mathbb{W}_{q} \times \mathbb{W}_{q}, \mathbb{W}_{q} \times \mathbb{W}_{q}\right)
$$

with partial Frechét derivatives at $(\eta, w, v)=(\eta, 0,0)$ given by

$$
F_{(w, v)}(\eta, 0,0)(\phi, \psi)=\left(\begin{array}{c}
\phi-T\left(-2 \alpha_{1} u_{\eta} \phi+\alpha_{2} u_{\eta} \psi, \eta \Phi\right) \\
\psi-T\left(\beta_{2} u_{\eta} \psi, \xi \Psi\right)
\end{array}\right)
$$

and

$$
F_{\eta,(w, v)}(\eta, 0,0)(\phi, \psi)=\left(\begin{array}{c}
-T\left(-2 \alpha_{1} u_{\eta}^{\prime} \phi+\alpha_{2} u_{\eta}^{\prime} \psi, \Phi\right) \\
-T\left(\beta_{2} u_{\eta}^{\prime} \psi, 0\right)
\end{array}\right)
$$

for $(\phi, \psi) \in \mathbb{W}_{q} \times \mathbb{W}_{q}$, where $u_{\eta}^{\prime}:=\frac{\partial}{\partial \eta} u_{\eta}$ is well defined according to Theorem A.4. We next show that the kernel of $F_{(w, v)}\left(\eta_{0}, 0,0\right)$ is one-dimensional. Given $(\phi, \psi) \in \operatorname{ker}\left(F_{(w, v)}\left(\eta_{0}, 0,0\right)\right)$ we have

$$
\begin{aligned}
\partial_{a} \phi-\Delta_{D} \phi & =-2 \alpha_{1} u_{\eta_{0}} \phi+\alpha_{2} u_{\eta_{0}} \psi, & \phi(0)=\eta_{0} \Phi, \\
\partial_{a} \psi-\Delta_{D} \psi & =\beta_{2} u_{\eta_{0}} \psi, & \psi(0)=\xi \Psi .
\end{aligned}
$$

From (3.8) and (3.4) we conclude that $\psi=\mu \psi_{*}$ for some $\mu \in \mathbb{R}$ with

$$
\psi_{*}:=\Pi_{\left[-\beta_{2} u_{\eta_{0}}\right]}(\cdot, 0) \Psi_{0} \in \mathbb{W}_{q} .
$$

Plugging this into 3.7 and observing that $1-\eta_{0} H_{\left[2 \alpha_{1} u_{\eta_{0}}\right]}$ is invertible since

$$
\eta_{0} r\left(H_{\left[2 \alpha_{1} u_{\eta_{0}}\right]}\right)<\eta_{0} r\left(H_{\left[\alpha_{1} u_{\eta_{0}}\right]}\right)=1
$$


by (2.9), Lemma A.3, and the positivity of $u_{\eta_{0}}$, we derive $\phi=\mu \phi_{*}$, where

$$
\phi_{*}:=\Pi_{\left[2 \alpha_{1} u_{\eta_{0}}\right]}(\cdot, 0) \Phi_{0}+S \psi_{*} \in \mathbb{W}_{q}, \quad a \in J,
$$

with

$$
\begin{gathered}
\left(S \psi_{*}\right)(a):=\alpha_{2} \int_{0}^{a} \Pi_{\left[2 \alpha_{1} u_{\eta_{0}}\right]}(a, \sigma)\left(u_{\eta_{0}}(\sigma) \psi_{*}(\sigma)\right) \mathrm{d} \sigma, \quad a \in J, \\
\Phi_{0}:=\eta_{0}\left(1-\eta_{0} H_{\left[2 \alpha_{1} u_{\eta_{0}}\right]}\right)^{-1} \int_{0}^{a_{m}} b_{1}(a)\left(S \psi_{*}\right)(a) \mathrm{d} a .
\end{gathered}
$$

Therefore,

$$
\operatorname{ker}\left(F_{(w, v)}\left(\eta_{0}, 0,0\right)\right)=\operatorname{span}\left\{\left(\phi_{*}, \psi_{*}\right)\right\} .
$$

As [3, Thm.1.1] and Sobolev's embedding theorem ensure the compact embedding $\mathbb{W}_{q} \hookrightarrow L_{\infty}(J, C(\bar{\Omega}))$ since $q>n+2$, we have

$$
\mathbb{W}_{q} \times \mathbb{W}_{q} \rightarrow \mathbb{L}_{q}, \quad(w, v) \mapsto w v \quad \text { is compact . }
$$

In particular, it readily follows that the derivative of $F$ has the form $F_{(w, v)}\left(\eta_{0}, 0,0\right)=1-\hat{T}$ with a compact operator $\hat{T}$. From this we get that also the codimension of $\operatorname{rg}\left(F_{(w, v)}\left(\eta_{0}, 0,0\right)\right)$ equals one. We next check the transversality condition of [10]. For, suppose that

$$
F_{\eta,(w, v)}\left(\eta_{0}, 0,0\right)\left(\phi_{*}, \psi_{*}\right) \in \operatorname{rg}\left(F_{(w, v)}\left(\eta_{0}, 0,0\right)\right) .
$$

Then there exists $v \in \mathbb{W}_{q}$ with

$$
v-T\left(\beta_{2} u_{\eta_{0}} v, \xi V\right)=-T\left(\beta_{2} u_{\eta_{0}}^{\prime} \psi_{*}, 0\right) .
$$

Choosing $\tau>0$ such that $\tau \Psi_{0}-v(0) \in \operatorname{int}\left(W_{q, D}^{2-2 / q,+}\right)$ and putting $p:=\tau \psi_{*}-v$, we obtain from the definition of $\psi_{*}$

$$
p(a)=\Pi_{\left[-\beta_{2} u_{\eta_{0}}\right]}(a, 0) p(0)+\int_{0}^{a} \Pi_{\left[-\beta_{2} u_{\eta_{0}}\right]}(a, \sigma)\left(\beta_{2} u_{\eta_{0}}^{\prime}(\sigma) \psi_{*}(\sigma)\right) \mathrm{d} \sigma, \quad a \in J .
$$

Thus, since $p(0)=\xi P$,

$$
\left(1-\xi \hat{H}_{\left[-\beta_{2} u_{\eta_{0}}\right]}\right) p(0)=\xi \int_{0}^{a_{m}} b_{2}(a) \int_{0}^{a} \Pi_{\left[-\beta_{2} u_{\eta_{0}}\right]}(a, \sigma)\left(\beta_{2} u_{\eta_{0}}^{\prime}(\sigma) \psi_{*}(\sigma)\right) \mathrm{d} \sigma \mathrm{d} a .
$$

However, as the right hand side belongs to $W_{q, D}^{2-2 / q,+} \backslash\{0\}$ due to 1.5), Theorem A.4, and the strong positivity of the evolution operator $\Pi_{\left[-\beta_{2} u_{\left.\eta_{0}\right]}\right.}(a, \sigma)$ on $W_{q, D}^{2-2 / q}$ for $0 \leqslant \sigma<a \leqslant a_{m}$, this last equation admits no positive solution $p(0)$ according to [1, Thm.3.2] and (3.3) which clearly contradicts the fact that $p(0)=\tau \Psi_{0}-v(0)$ belongs to $\operatorname{int}\left(W_{q, D}^{2-2 / q,+}\right)$. Consequently,

$$
F_{\eta,(w, v)}\left(\eta_{0}, 0,0\right)\left(\phi_{*}, \psi_{*}\right) \notin \operatorname{rg}\left(F_{(w, v)}\left(\eta_{0}, 0,0\right)\right) .
$$

We are thus in a position to apply [10, Thm.1.7] and deduce the existence of some $\varepsilon_{0}>0$ and functions $\eta \in C\left(\left(-\varepsilon_{0}, \varepsilon_{0}\right), \mathbb{R}\right)$ and $\theta_{j} \in C\left(\left(-\varepsilon_{0}, \varepsilon_{0}\right), \mathbb{W}_{q}\right)$ with $\eta(0)=\eta_{0}, \theta_{j}(0)=0$ such that the nontrivial zeros of the function $F$ close to $\left(\eta_{0}, 0,0\right)$ lie on the curve

$$
\left\{\left(\eta(\varepsilon), \varepsilon\left(\phi_{*}, \psi_{*}\right)+\varepsilon\left(\theta_{1}(\varepsilon), \theta_{2}(\varepsilon)\right)\right) ;|\varepsilon|<\varepsilon_{0}\right\} .
$$

By Theorem A.4

$$
\mathfrak{C}_{1}:=\left\{\left(\eta(\varepsilon), u_{\eta(\varepsilon)}+\varepsilon \phi_{*}+\varepsilon \theta_{1}(\varepsilon), \varepsilon \psi_{*}+\varepsilon \theta_{2}(\varepsilon)\right) ; 0<\varepsilon<\varepsilon_{0}\right\}
$$

is then a continuous curve of solutions to (1.11)-(1.12), (1.3)-(1.4) bifurcating from $\left(\eta_{0}, u_{\eta_{0}}, 0\right) \in \mathfrak{B}_{1}$. As all traces $u_{\eta_{0}}(0), \phi_{*}(0)=\Phi_{0}$, and $\psi_{*}(0)=\Psi_{0}$ belong to $\operatorname{int}\left(W_{q, D}^{2-2 / q,+}\right)$, it follows from (2.1) and the continuity of $\theta_{j}$ that the initial values $u(0)$ and $v(0)$ for a solution $(\eta, u, v) \in \mathfrak{C}_{1}$ belong to $\operatorname{int}\left(W_{q, D}^{2-2 / q,+}\right)$ provided $\varepsilon_{0}>0$ is sufficiently small, whence

$$
(u, v) \in \dot{\mathbb{W}}_{q}^{+} \times \dot{\mathbb{W}}_{q}^{+}, \quad(\eta, u, v) \in \mathfrak{C}_{1},
$$


by (2.5), (2.6), and positivity of the corresponding evolution operators. This completes the proof of the lemma.

Next we show that $\mathfrak{C}_{1}$ extends to a global continuum of positive coexistence solutions by invoking Rabinowitz' global alternative [23] along with the unilateral global results of López-Gómez [20]. The main steps of the proof are the same as in the proof of [29, Thm.2.2], but we have to argue here more subtle at several points since we are deriving bifurcation with respect to the parameter $\eta$ by linearizing around a point $\left(\eta, u_{\eta}, 0\right) \in \mathfrak{B}_{1}$.

Setting $u_{\eta}:=0$ for $\eta \leqslant 1$ it follows from Theorem A.4 that

$$
\left(\eta \mapsto u_{\eta}\right) \in C\left(\mathbb{R}, \mathbb{W}_{q}^{+}\right) .
$$

Hence, defining

$$
\begin{aligned}
& Z_{1}[\eta]:=\left(\partial_{a}-\Delta_{D}+2 \alpha_{1} u_{\eta}, \gamma_{0}\right)^{-1} \in \mathcal{L}\left(\mathbb{L}_{q} \times W_{q, D}^{2-2 / q}, \mathbb{W}_{q}\right), \\
& Z_{2}[\eta]:=\left(\partial_{a}-\Delta_{D}-\beta_{2} u_{\eta}, \gamma_{0}\right)^{-1} \in \mathcal{L}\left(\mathbb{L}_{q} \times W_{q, D}^{2-2 / q}, \mathbb{W}_{q}\right),
\end{aligned}
$$

based on maximal $L_{q}$-regularity (see Section 2 ), we deduce

$$
\left(\eta \mapsto Z_{j}[\eta]\right) \in C\left(\mathbb{R}, \mathcal{L}\left(\mathbb{L}_{q} \times W_{q, D}^{2-2 / q}, \mathbb{W}_{q}\right)\right), \quad j=1,2 .
$$

Writing again $(\eta, u, v)=\left(\eta, u_{\eta}+w, v\right) \in \mathbb{R} \times \mathbb{W}_{q} \times \mathbb{W}_{q}$ and recalling (3.5) and (3.6), it follows that (1.11)-(1.12) subject to (1.3)-(1.4) may be recast equivalently as

$$
(w, v)-K(\eta)(w, v)+R(\eta, w, v)=0
$$

by setting

$$
K(\eta)(w, v):=\left(\begin{array}{c}
Z_{1}[\eta]\left(\alpha_{2} u_{\eta} v, \eta W\right) \\
Z_{2}[\eta](0, \xi V)
\end{array}\right), \quad R(\eta, w, v):=-\left(\begin{array}{c}
Z_{1}[\eta]\left(-\alpha_{1} w^{2}+\alpha_{2} w v, 0\right) \\
Z_{2}[\eta]\left(-\beta_{1} v^{2}+\beta_{2} w v, 0\right)
\end{array}\right)
$$

for $(w, v) \in \mathbb{W}_{q} \times \mathbb{W}_{q}$ still using the notation

$$
W:=\int_{0}^{a_{m}} b_{1}(a) w(a) \mathrm{d} a, \quad V:=\int_{0}^{a_{m}} b_{2}(a) v(a) \mathrm{d} a .
$$

This notation we shall use throughout the remainder of this section as no confusion seems likely. Then (3.9), (3.10), 3.11), and the compact embedding $W_{q}^{2} \hookrightarrow W_{q, D}^{2-2 / q}$ entail

$$
K(\eta) \in \mathcal{K}\left(\mathbb{W}_{q} \times \mathbb{W}_{q}\right) \text { depends continuously on } \eta \in \mathbb{R},
$$

and

with

$$
R \in C\left(\mathbb{R} \times \mathbb{W}_{q} \times \mathbb{W}_{q}, \mathbb{W}_{q} \times \mathbb{W}_{q}\right) \text { is compact }
$$

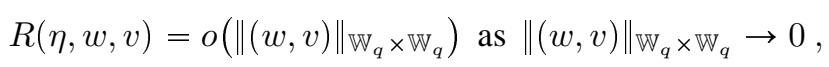

uniformly with respect to $\eta$ in compact intervals. Moreover, we have:

Lemma 3.3. Let $\eta \in \mathbb{R}$. If $\mu \geqslant 1$ is an eigenvalue of the compact operator $K(\eta)$ with eigenvector $(w, v) \in \mathbb{W}_{q} \times \mathbb{W}_{q}$, then either $\eta>1$ and $\mu / \xi$ is an eigenvalue of $\hat{H}_{\left[-\beta_{2} u_{\eta}\right]}$ with eigenvector $v(0) \in W_{q, D}^{2-2 / q}$ or $\mu=\eta=1$.

Proof. Let $\mu \geqslant 1$ and $(w, v) \in\left(\mathbb{W}_{q} \times \mathbb{W}_{q}\right) \backslash\{(0,0)\}$ with $K(\eta)(w, v)=\mu(w, v)$. On the one hand, if $v=0$, then

from which

$$
\partial_{a} w-\Delta_{D} w+2 \alpha_{1} u_{\eta} w=0, \quad w(0)=\frac{\eta}{\mu} W,
$$

$$
w(a)=\Pi_{\left[2 \alpha_{1} u_{\eta}\right]}(a, 0) w(0), \quad a \in J, \quad w(0)=\frac{\eta}{\mu} H_{\left[2 \alpha_{1} u_{\eta}\right]} w(0) .
$$


In particular, $\eta \neq 0$ and $w(0) \neq 0$ since otherwise $(w, v)=(0,0)$, and hence

$$
\mu \leqslant \operatorname{\eta r}\left(H_{\left[2 \alpha_{1} u_{\eta}\right]}\right) .
$$

Next, $\eta>1$ is impossible since otherwise $u_{\eta} \in \dot{\mathbb{W}}_{q}^{+}$and so

$$
\frac{\eta}{\mu} r\left(H_{\left[2 \alpha_{1} u_{\eta}\right]}\right)<\operatorname{\eta r}\left(H_{\left[\alpha_{1} u_{\eta}\right]}\right)=1
$$

by Lemma A.3 and (2.9) contradicting (3.17). Hence $\eta \leqslant 1$ and thus

$$
\frac{\eta}{\mu} r\left(H_{\left[2 \alpha_{1} u_{\eta}\right]}\right)=\frac{\eta}{\mu} r\left(H_{[0]}\right)=\frac{\eta}{\mu} \leqslant 1
$$

by (2.4) what is only possible if $\mu=\eta=1$ according to (3.17). On the other hand, if $v \neq 0$, then from

$$
\partial_{a} v-\Delta_{D} v-\beta_{2} u_{\eta} v=0, \quad v(0)=\frac{\xi}{\mu} V
$$

it follows

$$
v(a)=\Pi_{\left[-\beta_{2} u_{\eta}\right]}(a, 0) v(0), \quad a \in J, \quad v(0)=\frac{\xi}{\mu} \hat{H}_{\left[-\beta_{2} u_{\eta}\right]} v(0),
$$

and so $v(0) \neq 0$ and $\xi \neq 0$ since otherwise $v=0$. Consequently, $\mu / \xi$ is an eigenvalue of $\hat{H}_{\left[-\beta_{2} u_{\eta}\right]}$ with eigenvector $v(0)$. Assuming $\eta \leqslant 1$ we have $u_{\eta}=0$ and thus $\mu / \xi \leqslant r\left(\hat{H}_{[0]}\right)=1$ by (2.4) contradicting $0<\xi<1$ and $\mu \geqslant 1$.

As a consequence of Lemma 3.3 the set of singular values of the family $K(\eta)$ is discrete:

Corollary 3.4. The set $\Sigma:=\{\eta \in \mathbb{R} ; \operatorname{dim}(\operatorname{ker}(1-K(\eta))) \geqslant 1\}$ is discrete.

Proof. Lemma3.3 ensures

$$
\Sigma \cap(1, \infty) \subset \Xi:=\left\{\eta>1 ; \operatorname{dim}\left(\operatorname{ker}\left(1-\xi \hat{H}_{\left[-\beta_{2} u_{\eta}\right]}\right)\right) \geqslant 1\right\} .
$$

Due to

$$
\Pi_{\left[-\beta_{2} u_{\eta}\right]}(\cdot, 0) \Phi=\left(\partial_{a}-\Delta_{D}-\beta_{2} u_{\eta}, \gamma_{0}\right)^{-1}(0, \Phi), \quad \Phi \in W_{q, D}^{2-2 / q}, \quad \eta>1,
$$

it follows from the analyticity of the inversion map for linear operators and the analyticity of the map $\eta \mapsto u_{\eta}$ stated in Theorem A.4 that also the map $(1, \infty) \rightarrow \mathcal{K}\left(W_{q, D}^{2-2 / q}\right), \eta \mapsto \xi \hat{H}_{\left[-\beta_{2} u_{\eta}\right]}$ is real analytic. Thus, since $1-\xi \hat{H}_{\left[-\beta_{2} u_{\eta}\right]}$ is invertible for $\eta \in\left(1, \eta_{0}\right)$ owing to (3.1) and (3.3), we are in a position to apply [20, Thm.4.4.4] and conclude that $\Xi$ is discrete. If $\eta \in \Sigma$ with $\eta \leqslant 1$, then necessarily $\eta=1$ by Lemma 3.3

Next, we characterize the dependence on the parameter $\eta$ of the fixed point index $\operatorname{Ind}(0, K(\eta))$ of zero with respect to $K(\eta)$. Recall that $\operatorname{Ind}(0, K(\eta))=(-1)^{\zeta(\eta)}$, where $\zeta(\eta)$ is the sum of the algebraic multiplicities of all real eigenvalues of $K(\eta)$ greater than one, see e.g. [20, Sect.5.6].

Lemma 3.5. The fixed point index $\operatorname{Ind}(0, K(\eta))$ of zero with respect to $K(\eta)$ changes sign as $\eta$ crosses $\eta_{0}$.

Proof. First, let $1<\eta<\eta_{0}$ and suppose there is an eigenvalue $\mu>1$ of $K(\eta)$. Let $(w, v) \in \mathbb{W}_{q} \times \mathbb{W}_{q}$ be a corresponding eigenvector. Then Lemma 3.3 yields

$$
\mu \leqslant \xi r\left(\hat{H}_{\left[-\beta_{2} u_{\eta}\right]}\right) \text {. }
$$

Since $\eta<\eta_{0}$ we have $u_{\eta} \leqslant u_{\eta_{0}}$ owing to Theorem A.4. But then, by Lemma A.3, (3.18), and the assumption $\mu>1$,

$$
1<\xi r\left(\hat{H}_{\left[-\beta_{2} u_{\eta}\right]}\right)<\xi r\left(\hat{H}_{\left[-\beta_{2} u_{\eta_{0}}\right]}\right)
$$

in contradiction to the definition of $\eta_{0}$ in (3.3). Thus there is no eigenvalue $\mu>1$ of $K(\eta)$ if $1<\eta<\eta_{0}$, consequently

$$
\operatorname{Ind}(0, K(\eta))=1, \quad 1<\eta<\eta_{0} .
$$


Next, it follows from [2, II.Lem.5.1.4] and Theorem A.4 that the evolution operator $\Pi_{\left[-\beta_{2} u_{\eta}\right]}(\cdot, 0)$ depends continuously on $\eta$ (actually: analytically, cf. the proof of Corollary 3.4) and hence

$$
\hat{H}_{\left[-\beta_{2} u_{\eta}\right]} \longrightarrow \hat{H}_{\left[-\beta_{2} u_{\eta_{0}}\right]} \text { in } \mathcal{K}\left(W_{q, D}^{2-2 / q}\right) \text { as } \eta \longrightarrow \eta_{0} .
$$

According to [17, IV.§3.5],

$$
\lambda_{2}\left(\xi \hat{H}_{\left[-\beta_{2} u_{\eta}\right]}\right) \longrightarrow \lambda_{2}\left(\xi \hat{H}_{\left[-\beta_{2} u_{\eta_{0}}\right]}\right)<r\left(\xi \hat{H}_{\left[-\beta_{2} u_{\eta_{0}}\right]}\right)=1 \text { as } \eta \longrightarrow \eta_{0},
$$

with $\lambda_{2}(H)$ denoting the second eigenvalue of a compact operator $H$. Choose $\varepsilon>0$ with

$$
\lambda_{2}\left(\xi \hat{H}_{\left[-\beta_{2} u_{\eta}\right]}\right)<1-\varepsilon, \quad \eta_{0}<\eta<\eta_{0}+\varepsilon .
$$

Let $\eta_{0} \leqslant \eta<\eta_{0}+\varepsilon$ and let $\mu \geqslant 1$ be an eigenvalue of $K(\eta)$. Then $\mu \geqslant 1$ is an eigenvalue of $\xi \hat{H}_{\left[-\beta_{2} u_{\eta}\right]}$ due to Lemma 3.3 and thus $\mu=r\left(\xi \hat{H}_{\left[-\beta_{2} u_{\eta}\right]}\right)=: \mu_{*}$ since $\mu_{*}$ is the only eigenvalue in $(1-\varepsilon, \infty)$ by (3.19). But $\mu_{*}$ is a simple eigenvalue of $K(\eta)$. Indeed, noticing that

$$
K(\eta)(\phi, \psi)=\mu_{*}(\phi, \psi)
$$

is equivalent to

$$
\begin{aligned}
\partial_{a} \phi-\Delta_{D} \phi & =-2 \alpha_{1} u_{\eta} \phi+\frac{\alpha_{2}}{\mu_{*}} u_{\eta} \psi, & \phi(0) & =\frac{\eta}{\mu_{*}} \Phi, \\
\partial_{a} \psi-\Delta_{D} \psi & =\beta_{2} u_{\eta} \psi, & \psi(0) & =\frac{\xi}{\mu_{*}} \Psi,
\end{aligned}
$$

it follows as in the proof of Lemma 3.3 (see (3.7) and (3.8) that

$$
\operatorname{ker}\left(K(\eta)-\mu_{*}\right)=\operatorname{span}\left\{\left(\phi_{*}, \psi_{*}\right)\right\},
$$

where

$$
\psi_{*}:=Z_{2}[\eta]\left(0, \Psi_{1}\right)=\Pi_{\left[-\beta_{2} u_{\eta}\right]}(\cdot, 0) \Psi_{1} \in \dot{\mathbb{W}}_{q}^{+}
$$

with $\Psi_{1} \in \operatorname{int}\left(W_{q, D}^{2-2 / q,+}\right)$ spanning $\operatorname{ker}\left(\mu_{*}-\xi \hat{H}_{\left[-\beta_{2} u_{\eta}\right]}\right)$ and

$$
\begin{aligned}
\Phi_{1} & :=\frac{\eta}{\mu_{*}}\left(1-\frac{\eta}{\mu_{*}} H_{\left[2 \alpha_{1} u_{\eta}\right]}\right)^{-1} \int_{0}^{a_{m}} b_{1}(a)\left(S \psi_{*}\right)(a) \mathrm{d} a \in W_{q, D}^{2-2 / q,+}, \\
\phi_{*} & :=\Pi_{\left[2 \alpha_{1} u_{\eta}\right]}(\cdot, 0) \Phi_{1}+S \psi_{*}=Z_{1}[\eta]\left(S \psi_{*}, \Phi_{1}\right) \in \mathbb{W}_{q}^{+} .
\end{aligned}
$$

Invertibility of $1-\frac{\eta}{\mu_{*}} H_{\left[2 \alpha_{1} u_{\eta}\right]}$ is due to $\mu_{*} \geqslant 1,[2.9]$, and Lemma A.3 It then merely remains to prove that $\mu_{*}$ is simple. For, let $\left(\phi_{*}, \psi_{*}\right) \in \operatorname{rg}\left(K(\eta)-\mu_{*}\right)$. Then

$$
Z_{2}[\eta](0, \xi V)-\mu_{*} v=\psi_{*}
$$

for some $v \in \mathbb{W}_{q}$, that is,

$$
\partial_{a} v-\Delta_{D} v-\beta_{2} u_{\eta} v=-\frac{1}{\mu_{*}}\left(\partial_{a} \psi_{*}-\Delta_{D} \psi_{*}-\beta_{2} u_{\eta} \psi_{*}\right)=0, \quad v(0)=\frac{\xi}{\mu_{*}} V-\frac{1}{\mu_{*}} \Psi_{1} .
$$

This readily implies

so that

$$
\left(1-\frac{\xi}{\mu_{*}} \hat{H}_{\left[-\beta_{2} u_{\eta}\right]}\right) v(0)=-\frac{1}{\mu_{*}} \Psi_{1}
$$

$$
\Psi_{1} \in \operatorname{ker}\left(1-\frac{\xi}{\mu_{*}} \hat{H}_{\left[-\beta_{2} u_{\eta}\right]}\right) \cap \operatorname{rg}\left(1-\frac{\xi}{\mu_{*}} \hat{H}_{\left[-\beta_{2} u_{\eta}\right]}\right)
$$

contradicting the fact that the intersection equals $\{0\}$ since $\mu_{*} / \xi=r\left(\hat{H}_{\left[-\beta_{2} u_{\eta}\right]}\right)$ is a simple eigenvalue of $\hat{H}_{\left[-\beta_{2} u_{\eta}\right]}$. Thus $\left(\phi_{*}, \psi_{*}\right) \notin \operatorname{rg}\left(K(\xi)-\mu_{*}\right)$ and $\mu_{*}$ is indeed a simple eigenvalue of $K(\eta)$. This ensures

$$
\operatorname{Ind}(0, K(\eta))=-1, \quad \eta_{0} \leqslant \eta<\eta_{0}+\varepsilon,
$$

and the assertion follows. 
Recalling the definition of $\Psi_{0}$ in (3.4) and taking $\eta=\eta_{0}$ (and so $\mu_{*}=1$ ), the proof of Lemma 3.5 reveals:

Corollary 3.6. $\mu_{\star}=1$ is a simple eigenvalue of $K\left(\eta_{0}\right)$. Thus

$$
\mathbb{W}_{q} \times \mathbb{W}_{q}=\operatorname{ker}\left(1-K\left(\eta_{0}\right)\right) \oplus \operatorname{rg}\left(1-K\left(\eta_{0}\right)\right), \quad \operatorname{ker}\left(1-K\left(\eta_{0}\right)\right)=\operatorname{span}\left\{\left(\phi_{\star}, \psi_{\star}\right)\right\}
$$

with $\psi_{\star}=Z_{2}\left[\eta_{0}\right]\left(0, \Psi_{0}\right) \in \dot{\mathbb{W}}_{q}^{+}, \Psi_{0}=\xi \Psi_{\star} \in \operatorname{int}\left(W_{q, D}^{2-2 / q,+}\right)$, and $\phi_{\star} \in \dot{\mathbb{W}}_{q}^{+}$.

Corollary 3.4 and Lemma 3.5 warrant that we may apply Rabinowitz' global alternative [20, Cor.6.3.2] to (3.12). Hence, we obtain a continuum $\mathfrak{C}_{1}^{\prime}$ of solutions $(\eta, u, v)$ in $\mathbb{R} \times \mathbb{W}_{q} \times \mathbb{W}_{q}$ to (1.11)-(1.12) subject to (1.3)-(1.4) emanating from $\left(\eta_{0}, u_{\eta_{0}}, 0\right) \in \mathfrak{B}_{1}$. In combination with the unilateral global bifurcation result [20, Thm.6.4.3] and Corollary 3.6 we derive that $\mathfrak{C}_{1}^{\prime}$ satisfies the alternatives

(i) $\mathfrak{C}_{1}^{\prime}$ is unbounded in $\mathbb{R} \times \mathbb{W}_{q} \times \mathbb{W}_{q}$, or

(ii) there is $\eta \in \Sigma \backslash\left\{\eta_{0}\right\}$ with $\left(\eta, u_{\eta}, 0\right) \in \mathfrak{C}_{1}^{\prime}$, or

(iii) there is $\left(\eta, u_{\eta}+w, v\right) \in \mathfrak{C}_{1}^{\prime}$ with $(w, v) \in \operatorname{rg}\left(1-K\left(\eta_{0}\right)\right) \backslash\{(0,0)\}$.

By Lemma 3.2. $\mathfrak{C}_{1}^{\prime}$ close to $\left(\eta_{0}, u_{\eta_{0}}, 0\right)$ coincides with $\mathfrak{C}_{1} \subset \mathbb{R}^{+} \times \dot{\mathbb{W}}_{q}^{+} \times \dot{\mathbb{W}}_{q}^{+}$suggesting to abuse notation by putting

In fact, we have:

$$
\mathfrak{C}_{1}:=\mathfrak{C}_{1}^{\prime \prime} \cap\left(\mathbb{R}^{+} \times \dot{\mathbb{W}}_{q}^{+} \times \dot{\mathbb{W}}_{q}^{+}\right) \neq \varnothing
$$

Lemma 3.7. $\mathfrak{C}_{1}$ is unbounded in $\mathbb{R}^{+} \times \dot{\mathbb{W}}_{q}^{+} \times \dot{\mathbb{W}}_{q}^{+}$.

Proof. Suppose $\mathfrak{C}_{1}^{\prime}$ leaves $\mathbb{R}^{+} \times \dot{\mathbb{W}}_{q}^{+} \times \dot{\mathbb{W}}_{q}^{+}$at some point $(\eta, u, v) \in \mathfrak{C}_{1}^{\prime}$ different from $\left(\eta_{0}, u_{\eta_{0}}, 0\right)$ and let $\left(\eta_{j}, u_{j}, v_{j}\right) \in \mathfrak{C}_{1}$ such that

$$
\left(\eta_{j}, u_{j}, v_{j}\right) \longrightarrow(\eta, u, v) \quad \text { in } \quad \mathbb{R} \times \mathbb{W}_{q} \times \mathbb{W}_{q} .
$$

Since obviously $\eta \geqslant 0, u \geqslant 0$, and $v \geqslant 0$, the only possibility is that $u=0$ or $v=0$. However, as the only solutions in $\mathbb{R}^{+} \times \mathbb{W}_{q}^{+} \times \mathbb{W}_{q}^{+}$close to $\mathfrak{B}_{0}$ lie on $\mathfrak{B}_{1}$, the case $(u, v)=(0,0)$ is impossible since $v_{j} \neq 0$. If $u=0$ but $v \neq 0$, then $(\eta, u, v)=(\eta, 0, v) \in \mathfrak{C}_{1}^{\prime}$ and $v \in \dot{\mathbb{W}}_{q}^{+}$solves

$$
\partial_{a} v-\Delta_{D} v=-\beta_{1} v^{2}, \quad v(0)=\xi V
$$

with $\xi<1$ contradicting Theorem A.4 Consequently, $v=0$ but $u \neq 0$, thus $u \in \dot{\mathbb{W}}_{q}^{+}$solves

$$
\partial_{a} u-\Delta_{D} u=-\alpha_{1} u^{2}, \quad u(0)=\eta U,
$$

whence $\eta>1$ and $u=u_{\eta}$ by Theorem $\mathrm{A} .4$ Therefore, $(\eta, u, v)=\left(\eta, u_{\eta}, 0\right) \in \mathfrak{C}_{1}^{\prime \prime}$ and we may assume that $\eta_{j}>1$. To demonstrate that this also leads to a contradiction, we adapt an argument of [5, Thm.3.1]. Put $z_{j}:=\left(w_{j}, v_{j}\right)$, where $w_{j}:=u_{j}-u_{\eta_{j}}$, and note that $z_{j} \rightarrow(0,0)$ as $j \rightarrow \infty$ by the previous observation. Moreover, since $u_{j}, v_{j} \geqslant 0$ and $\eta_{j}>1$, we obtain from

$$
\partial_{a} u_{j}-\Delta_{D} u_{j}=-\alpha_{1} u_{j}^{2}+\alpha_{2} v_{j} u_{j} \geqslant-\alpha_{1} u_{j}^{2}, \quad u_{j}(0)=\eta_{j} U_{j},
$$

that $u_{j} \geqslant u_{\eta_{j}}$ by invoking Lemma A.3, whence $z_{j} \in \mathbb{W}_{q}^{+} \times \mathbb{W}_{q}^{+}$. We then define

$$
Q: \mathbb{R} \times \mathbb{W}_{q}^{2} \rightarrow \mathbb{W}_{q}^{2}, \quad Q(\zeta, z):=K(\zeta) z-R(\zeta, z)
$$

and observe that $Q$ is differentiable with respect to $z \in \mathbb{W}_{q}^{2}, Q(\zeta, 0)=0$ for $\zeta \in \mathbb{R}$, and $Q\left(\eta_{j}, z_{j}\right)=z_{j}$. The mean value theorem ensures

$$
z_{j}-Q_{z}(\eta, 0) z_{j}=\int_{0}^{1}\left[Q_{z}\left(\eta_{j}, s z_{j}\right) z_{j}-Q_{z}(\eta, 0) z_{j}\right] \mathrm{d} s
$$

and hence, setting $m_{j}:=z_{j} /\left\|z_{j}\right\|_{\mathbb{W}_{q}^{2}} \in \mathbb{W}_{q}^{+} \times \mathbb{W}_{q}^{+}$and taking $Q_{z}(\eta, 0)=K(\eta)$ into account,

$$
m_{j}-K(\eta) m_{j}=\int_{0}^{1}\left[Q_{z}\left(\eta_{j}, s z_{j}\right) m_{j}-Q_{z}(\eta, 0) m_{j}\right] \mathrm{d} s \longrightarrow 0 \text { as } j \rightarrow \infty
$$


by the boundedness of $\left(m_{j}\right)_{j \in \mathbb{N}},\left(\eta_{j}, z_{j}\right) \rightarrow(\eta, 0)$, and Lebesgue's theorem. As $K(\eta)$ is compact, this readily implies the existence of $m \in \mathbb{W}_{q}^{+} \times \mathbb{W}_{q}^{+}$with $\|m\|_{\mathbb{W}_{q}^{2}}=1$ and $m=K(\eta) m$. Owing to Lemma 3.3 we conclude that $1 / \xi$ is an eigenvalue of $\hat{H}_{\left[-\beta_{2} u_{\eta}\right]}$ with positive eigenvector. Hence $1=\xi r\left(\hat{H}_{\left[-\beta_{2} u_{\eta}\right]}\right)$ due to the Krein-Rutman theorem (see Lemma A.2) yielding $\eta=\eta_{0}$ what is impossible since $(\eta, u, v)$ then coincides with $\left(\eta_{0}, u_{\eta_{0}}, 0\right)$. Therefore, $\mathfrak{C}_{1}=\mathfrak{C}_{1}^{\prime}$ does not leave $\mathbb{R}^{+} \times \dot{\mathbb{W}}_{q}^{+} \times \dot{\mathbb{W}}_{q}^{+}$except at $\left(\eta_{0}, u_{\eta_{0}}, 0\right)$.

As a consequence of the preceding observation, alternative (ii) above can be ruled out. Suppose then that alternative (iii) above occurs, i.e. let $\left(\eta, u_{\eta}+w, v\right) \in \mathfrak{C}_{1}^{\prime}$ be such that

$$
(0,0) \neq(w, v)=\left(1-K\left(\eta_{0}\right)\right)(f, g)
$$

for some $(f, g) \in \mathbb{W}_{q} \times \mathbb{W}_{q}$. To derive a contradiction we argue similarly as in the proof of Lemma3.2 As $v \in \dot{\mathbb{W}}_{q}^{+}$, we have $v(0)=\xi V \in W_{q, D}^{2-2 / q,+} \backslash\{0\}$. Recall $\psi_{\star}(0)=\Psi_{0} \in \operatorname{int}\left(W_{q, D}^{2-2 / q,+}\right)$ from Corollary 3.6 so that we may choose $\tau>0$ with

$$
g(0)-v(0)+\tau \Psi_{0} \in \operatorname{int}\left(W_{q, D}^{2-2 / q,+}\right) .
$$

Note that

$$
v=g-Z_{2}\left[\eta_{0}\right](0, \xi G), \quad \psi_{\star}=Z_{2}\left[\eta_{0}\right]\left(0, \xi \Psi_{\star}\right), \quad p:=g-v+\tau \psi_{\star}=Z_{2}\left[\eta_{0}\right]\left(0, \xi\left(G+\tau \Psi_{\star}\right)\right) .
$$

The last equality reads

$$
\partial_{a} p-\Delta_{D} p-\beta_{2} u_{\eta_{0}} p=0, \quad p(0)=\xi\left(G+\tau \Psi_{\star}\right)=\xi P+\xi V,
$$

from which we deduce that

$$
\left(1-\xi \hat{H}_{\left[-\beta_{2} u_{\eta_{0}}\right]}\right) p(0)=\xi V \in W_{q, D}^{2-2 / q,+} \backslash\{0\}
$$

with $p(0) \in \operatorname{int}\left(W_{q, D}^{2-2 / q,+}\right)$ by the choice of $\tau$. However, this equation has no positive solution owing to [1, Thm.3.2] and the definition of $\xi$ in (3.3). This shows that alternative (iii) above is impossible as well and the only remaining possibility is that $\mathfrak{C}_{1}=\mathfrak{C}_{1}^{\prime}$ is unbounded in $\mathbb{R}^{+} \times \dot{\mathbb{W}}_{q}^{+} \times \dot{\mathbb{W}}_{q}^{+}$.

We remark that the bifurcation point $\left(\eta_{0}, u_{\eta_{0}}, 0\right)$ is unique:

Corollary 3.8. There is no other bifurcation point on $\mathfrak{B}_{1}$ to positive coexistence solutions than $\left(\eta_{0}, u_{\eta_{0}}, 0\right)$.

Proof. Suppose $\left(\eta, u_{\eta}, 0\right) \in \mathfrak{B}_{1}$ is a bifurcation point to positive coexistence solutions. Approximating this point by positive solutions we derive as in the proof of Lemma 3.7 that 1 is an eigenvalue of $K(\eta)$ with an eigenvector in $\mathbb{W}_{q}^{+} \times \mathbb{W}_{q}^{+}$so that, according to Lemma 3.3, $1 / \xi$ is an eigenvalue of $\hat{H}_{\left[-\beta_{2} u_{\eta}\right]}$ with positive eigenvector. As above, this implies $1=\xi r\left(\hat{H}_{\left[-\beta_{2} u_{\eta}\right]}\right)$ due to the Krein-Rutman theorem (see Lemma A.2), whence $\eta=\eta_{0}$.

This completes the proof of Theorem 1.1. It remains to give a more precise characterization of the global nature of $\mathfrak{C}_{1}$ as stated in Corollary 1.3 .

Corollary 3.9. The continuum $\mathfrak{C}_{1}$ is unbounded with respect to both the parameter $\eta$ and the u-component in $\mathbb{W}_{q}$, or with respect to the $v$-component in $\mathbb{W}_{q}$. If 1.13 holds for some $s>0$, then $\mathfrak{C}_{1}$ is unbounded with respect to the u-component in $\mathbb{W}_{q}$.

Proof. (i) We have $u \geqslant u_{\eta}$ for any $(\eta, u, v) \in \mathfrak{C}_{1}$ with $\eta>1$ by the comparison principle of Lemma A.1 since

$$
\partial_{a} u-\Delta_{D} u=-\alpha_{1} u^{2}+\alpha_{2} v u \geqslant-\alpha_{1} u^{2}, \quad u(0)=\eta U .
$$

Since $\left\|u_{\eta}(0)\right\|_{\infty} \rightarrow \infty$ as $\eta \rightarrow \infty$ according to Theorem $\mathrm{A}$.4 we conclude that $\mathfrak{C}_{1}$ is unbounded with respect to $\eta$ only if it is unbounded with respect to the $u$-component in $\mathbb{W}_{q}$.

(ii) Next suppose (1.13) and that there is $M>s / \beta_{2}$ such that $\|u(a)\|_{\infty} \leqslant M, a \in J$, for all $(\eta, u, v) \in \mathfrak{C}_{1}$. Noticing

$$
\partial_{a} v-\Delta_{D} v=-\beta_{1} v^{2}+\beta_{2} u v \leqslant-\beta_{1} v^{2}+\beta_{2} M v, \quad a \in\left(0, a_{m}\right), \quad x \in \Omega,
$$


it follows from the parabolic maximum principle [14, Thm.13.5] that $v(a) \leqslant f(a)$ on $\bar{\Omega}$ for $a \in J$, where

$$
f(a):=m\|v(0)\|_{\infty}\left(\beta_{1}\|v(0)\|_{\infty}\left(1-e^{-m a}\right)+m e^{-m a}\right)^{-1}, \quad a \in J,
$$

with $m:=\beta_{2} M>s$ satisfies

$$
f^{\prime}(a)=-\beta_{1} f^{2}(a)+m f(a), \quad a \in J, \quad f(0)=\|v(0)\|_{\infty} .
$$

Thus (1.4) and (1.13) imply

$$
v(0)=\xi V \leqslant \xi \int_{0}^{a_{m}} b_{2}(a) f(a) \mathrm{d} a \leqslant \frac{\xi m}{\beta_{1}} \int_{0}^{a_{m}} b_{2}(a)\left(1-e^{-s a}\right)^{-1} \mathrm{~d} a<\infty \quad \text { on } \bar{\Omega},
$$

and so, owing to the definition of $f$, there is some $c>0$ such that $\|v(a)\|_{\infty} \leqslant c, a \in J$ for all $(\eta, u, v) \in \mathfrak{C}_{1}$. Hence, $\mathfrak{C}_{1}$ is bounded with respect to the $v$-component by Lemma 2.1 contradicting our findings in (i). Consequently, if $(1.13)$ holds, then $\mathfrak{C}_{1}$ is unbounded with respect to the $u$-component in $\mathbb{W}_{q}$.

\section{Cooperative Systems With $\xi>1$ : Proof of Theorem 1.2}

We still focus our attention on (1.11)-(1.12) subject to (1.3)-(1.4), but let now $\xi>1$ be arbitrarily fixed for the remainder of this section and put

$$
\eta_{1}:=\eta_{1}(\xi):=\frac{1}{r\left(H_{\left[-\alpha_{2} v_{\xi}\right]}\right)} .
$$

Then $\eta_{1} \in(0,1)$ according to (2.4) and Lemma A.3. The Krein-Rutman theorem ensures

$$
\operatorname{ker}\left(1-\eta_{1} H_{\left[-\alpha_{2} v_{\xi}\right]}\right)=\operatorname{span}\left\{\Phi^{0}\right\} \quad \text { with } \quad \Phi^{0} \in \operatorname{int}\left(W_{q, D}^{2-2 / q,+}\right) .
$$

We first prove local bifurcation of a continuous curve from $\left(\eta_{1}(\xi), 0, v_{\xi}\right) \in \mathfrak{B}_{2}$ by invoking the theorem of Crandall-Rabinowitz [10]. The present situation, however, turns out to be simpler than in the previous section.

Lemma 4.1. A local continuous curve $\mathfrak{C}_{2}$ of positive coexistence solutions to (1.11)-(1.12) subject to (1.3)(1.4) bifurcates from $\left(\eta_{1}(\xi), 0, v_{\xi}\right) \in \mathfrak{B}_{2}$, and all positive coexistence solutions near $\left(\eta_{1}(\xi), 0, v_{\xi}\right)$ lie on this curve.

Proof. We proceed similar to the proof of Lemma 3.2. Writing solutions to (1.11)-(1.12) subject to (1.3)(1.4) in the form $(\eta, u, v)=\left(\eta, u, v_{\xi}+w\right) \in \mathbb{R} \times \mathbb{W}_{q} \times \mathbb{W}_{q}$, we have

$$
\begin{array}{lrl}
\partial_{a} u-\Delta_{D} u=-\alpha_{1} u^{2}+\alpha_{2} u\left(v_{\xi}+w\right), & u(0)=\eta U, \\
\partial_{a} w-\Delta_{D} w=-\beta_{1} w^{2}-2 \beta_{1} v_{\xi} w+\beta_{2} u\left(v_{\xi}+w\right), & w(0)=\xi W,
\end{array}
$$

where we agree upon the notation (and similarly for other capital letters)

$$
U:=\int_{0}^{a_{m}} b_{1}(a) u(a) \mathrm{d} a, \quad W:=\int_{0}^{a_{m}} b_{2}(a) w(a) \mathrm{d} a .
$$

Thus we are lead to examine the zeros of the function $G \in C^{2}\left((1, \infty) \times \mathbb{W}_{q} \times \mathbb{W}_{q}, \mathbb{W}_{q} \times \mathbb{W}_{q}\right)$ given by

$$
G(\eta, u, w):=\left(\begin{array}{c}
u-T\left(-\alpha_{1} u^{2}+\alpha_{2} u\left(v_{\xi}+w\right), \eta U\right) \\
w-T\left(-\beta_{1} w^{2}-2 \beta_{1} v_{\xi} w+\beta_{2} u\left(v_{\xi}+w\right), \xi W\right)
\end{array}\right),
$$

with $T$ as in the proof of Lemma3.2 For the partial Frechét derivatives at $(\eta, u, w)=(\eta, 0,0)$ we compute

$$
G_{(u, w)}(\eta, 0,0)(\phi, \psi)=\left(\begin{array}{c}
\phi-T\left(\alpha_{2} \phi v_{\xi}, \eta \Phi\right) \\
\psi-T\left(-2 \beta_{1} v_{\xi} \psi+\beta_{2} \phi v_{\xi}, \xi \Psi\right)
\end{array}\right)
$$

and

$$
G_{\eta,(u, w)}(\eta, 0,0)(\phi, \psi)=\left(\begin{array}{c}
-T(0, \Phi) \\
0
\end{array}\right)
$$


for $(\phi, \psi) \in \mathbb{W}_{q} \times \mathbb{W}_{q}$. Arguments similar to the ones in the proof of Lemma3.2 yield

$$
\operatorname{ker}\left(G_{(u, w)}\left(\eta_{1}, 0,0\right)\right)=\operatorname{span}\left\{\left(\phi_{*}, \psi_{*}\right)\right\},
$$

where (see (4.2) and Lemma A.3)

$$
\phi_{*}:=\Pi_{\left[-\alpha_{2} v_{\xi}\right]}(\cdot, 0) \Phi^{0} \in \mathbb{W}_{q}^{+},
$$

and

$$
\psi_{*}:=\Pi_{\left[2 \beta_{1} v_{\xi}\right]}(\cdot, 0) \Psi^{0}+S \phi_{*} \in \mathbb{W}_{q}, \quad \Psi^{0}:=\xi\left(1-\xi \hat{H}_{\left[2 \beta_{1} v_{\xi}\right]}\right)^{-1} \int_{0}^{a_{m}} b_{2}(a)\left(S \phi_{*}\right)(a) \mathrm{d} a,
$$

with

$$
\left(S \phi_{*}\right)(a):=\beta_{2} \int_{0}^{a} \Pi_{\left[2 \beta_{1} v_{\xi}\right]}(a, \sigma)\left(v_{\xi}(\sigma) \phi_{*}(\sigma)\right) \mathrm{d} \sigma, \quad a \in J .
$$

Observing that the derivative of $G$ has the form $G_{(u, w)}\left(\eta_{1}, 0,0\right)=1-\hat{T}$ with a compact operator $\hat{T}$ (see (3.9)), we get that also the codimension of $\operatorname{rg}\left(G_{(u, w)}\left(\eta_{1}, 0,0\right)\right)$ equals one. Next assume that

$$
G_{\eta,(u, w)}\left(\eta_{1}, 0,0\right)\left(\phi_{*}, \psi_{*}\right) \in \operatorname{rg}\left(G_{(u, w)}\left(\eta_{1}, 0,0\right)\right)
$$

and let $u \in \mathbb{W}_{q}$ be with

$$
u-T\left(\alpha_{2} v_{\xi} u, \eta_{1} U\right)=-T\left(0, \Phi_{*}\right) .
$$

Then

$$
\partial_{a} u-\Delta_{D} u=\alpha_{2} v_{\xi} u, \quad a \in J, \quad u(0)=\eta_{1} U-\Phi_{*} .
$$

This readily implies

$$
\Phi_{*}=-\left(1-\eta_{1} H_{\left[-\alpha_{2} v_{\xi}\right]}\right) u(0) \in \operatorname{rg}\left(1-\eta_{1} H_{\left[-\alpha_{2} v_{\xi}\right]}\right)
$$

in contradiction to

$$
\Phi_{*}=\frac{1}{\eta_{1}} \Phi^{0} \in \operatorname{ker}\left(1-\eta_{1} H_{\left[-\alpha_{2} v_{\xi}\right]}\right)
$$

by (4.2) and (4.5) since $\eta_{1} r\left(H_{\left[-\alpha_{2} v_{\xi}\right]}\right)=1$ is a simple eigenvalue of the compact operator $\eta_{1} H_{\left[-\alpha_{2} v_{\xi}\right]}$. Consequently,

$$
G_{\eta,(u, w)}\left(\eta_{1}, 0,0\right)\left(\phi_{*}, \psi_{*}\right) \notin \operatorname{rg}\left(G_{(u, w)}\left(\eta_{1}, 0,0\right)\right),
$$

and we may again apply [10, Thm.1.7]. Thus, the nontrivial zeros of the function $G$ lie on the curve

$$
\left\{\left(\eta(\varepsilon), \varepsilon\left(\phi_{*}, \psi_{*}\right)+\varepsilon\left(\theta_{1}(\varepsilon), \theta_{2}(\varepsilon)\right)\right) ;|\varepsilon|<\varepsilon_{0}\right\},
$$

for some $\varepsilon_{0}>0$ and functions $\eta \in C\left(\left(-\varepsilon_{0}, \varepsilon_{0}\right), \mathbb{R}\right)$ and $\theta_{j} \in C\left(\left(-\varepsilon_{0}, \varepsilon_{0}\right), \mathbb{W}_{q}\right)$ with $\eta(0)=\eta_{1}, \theta_{j}(0)=0$. Thus,

$$
\mathfrak{C}_{2}:=\left\{\left(\eta(\varepsilon), \varepsilon \phi_{*}+\varepsilon \theta_{1}(\varepsilon), v_{\xi}+\varepsilon \psi_{*}+\varepsilon \theta_{2}(\varepsilon)\right) ; 0<\varepsilon<\varepsilon_{0}\right\}
$$

defines a continuous curve of solutions to (1.11)-(1.12), (1.3)-(1.4) bifurcating from $\left(\eta_{1}, 0, v_{\xi}\right) \in \mathfrak{B}_{2}$. As $\phi_{*}(0)=\Phi^{0} \in \operatorname{int}\left(W_{q, D}^{2-2 / q,+}\right)$ and $v_{\xi}(0) \in \operatorname{int}\left(W_{q, D}^{2-2 / q,+}\right)$, it follows from (2.5) and (2.6) that

$$
(u, v) \in \dot{\mathbb{W}}_{q}^{+} \times \dot{\mathbb{W}}_{q}^{+}, \quad(\eta, u, v) \in \mathfrak{C}_{2},
$$

provided $\varepsilon_{0}>0$ is sufficiently small. This completes the proof of the lemma.

To prove the assertion on global bifurcation of Theorem 1.2 we invoke Rabinowitz' global alternative [23] and the unilateral global theorem [20] as in the proof of Theorem 1.1] Again, the present situation is considerably simpler than in the proof of Theorem 1.1 .

Lemma 4.2. The local curve $\mathfrak{C}_{2}$ extends to an unbounded continuum of coexistence solutions $(\eta, u, v)$ in $\mathbb{R}^{+} \times \dot{\mathbb{W}}_{q}^{+} \times \dot{\mathbb{W}}_{q}^{+}$to (1.11)-(1.12) subject to (1.3)-(1.4). 
Proof. Introducing the operators

$$
\begin{aligned}
& \tilde{Z}_{1}:=\left(\partial_{a}-\Delta_{D}-\alpha_{2} v_{\xi}, \gamma_{0}\right)^{-1} \in \mathcal{L}\left(\mathbb{L}_{q} \times W_{q, D}^{2-2 / q}, \mathbb{W}_{q}\right), \\
& \tilde{Z}_{2}:=\left(\partial_{a}-\Delta_{D}+2 \beta_{1} v_{\xi}, \gamma_{0}\right)^{-1} \in \mathcal{L}\left(\mathbb{L}_{q} \times W_{q, D}^{2-2 / q}, \mathbb{W}_{q}\right),
\end{aligned}
$$

we may rewrite (4.3)-(4.4) equivalently as

$$
(u, w)-\tilde{K}(\eta)(u, w)+\tilde{R}(u, w)=0
$$

by setting

$$
\tilde{K}(\eta)(u, w):=\left(\begin{array}{c}
\tilde{Z}_{1}(0, \eta U) \\
\tilde{Z}_{2}\left(\beta_{2} u v_{\xi}, \xi W\right)
\end{array}\right), \quad \tilde{R}(u, w):=-\left(\begin{array}{c}
\tilde{Z}_{1}\left(\alpha_{1} u^{2}+\alpha_{2} u w, 0\right) \\
\tilde{Z}_{2}\left(-\beta_{1} w^{2}+\beta_{2} u w, 0\right)
\end{array}\right)
$$

for $(u, w) \in \mathbb{W}_{q} \times \mathbb{W}_{q}$. It is now easy to check on the basis of the previous section that the analogues of (3.14), 3.15), 3.16, and accordingly Lemma 3.3, Corollary 3.4 Lemma 3.5, and Corollary 3.6 hold for $\tilde{K}$ and $\tilde{R}$ when replacing $\eta_{0}$ by $\eta_{1}$. Consequently, we may apply the results [20, Cor.6.3.2,Lem.6.4.1,Thm.6.4.3] on unilateral global bifurcation to (4.6) and thus derive the existence of a continuum $\mathfrak{C}_{2}^{\prime}$ of solutions $(\eta, u, v)$ to (1.11)-(1.12) subject to (1.3)-(1.4) in $\mathbb{R} \times \mathbb{W}_{q} \times \mathbb{W}_{q}$ emanating from $\left(\eta_{1}, 0, v_{\xi}\right)$ and satisfying the alternatives

(i) $\mathfrak{C}_{2}^{\prime}$ is unbounded in $\mathbb{R} \times \mathbb{W}_{q} \times \mathbb{W}_{q}$, or

(ii) $\mathfrak{C}_{2}^{\prime}$ contains a point $\left(\eta, 0, v_{\xi}\right)$ with $\eta \in\{\zeta \in \mathbb{R} ; \operatorname{dim}(\operatorname{ker}(1-\tilde{K}(\zeta))) \geqslant 1\}$ and $\eta \neq \eta_{1}$, or

(iii) $\mathfrak{C}_{2}^{\prime}$ contains a point $\left(\eta, u, v_{\xi}+w\right)$ with $(u, w) \in \operatorname{rg}\left(1-\tilde{K}\left(\eta_{1}\right)\right)$ with $(u, w) \neq(0,0)$.

By Lemma 4.1, $\mathfrak{C}_{2}^{\prime}$ coincides with $\mathfrak{C}_{2}$ near $\left(\eta_{1}, 0, v_{\xi}\right)$ suggesting to abuse notation by putting

$$
\mathfrak{C}_{2}:=\mathfrak{C}_{2}^{\prime} \cap\left(\mathbb{R}^{+} \times \dot{\mathbb{W}}_{q}^{+} \times \dot{\mathbb{W}}_{q}^{+}\right) \neq \varnothing \text {. }
$$

We then claim that this so defined continuum $\mathfrak{C}_{2}$ is unbounded in $\mathbb{R}^{+} \times \dot{\mathbb{W}}_{q}^{+} \times \dot{\mathbb{V}}_{q}^{+}$. Indeed, suppose $\mathfrak{C}_{2}^{\prime}$ leaves $\mathbb{R}^{+} \times \dot{\mathbb{V}}_{q}^{+} \times \dot{\mathbb{W}}_{q}^{+}$at some point $(\eta, u, v) \in \mathfrak{C}_{2}^{\prime}$ different from $\left(\eta_{1}, 0, v_{\xi}\right)$ and let $\left(\eta_{j}, u_{j}, v_{j}\right) \in \mathfrak{C}_{2}$ such that

$$
\left(\eta_{j}, u_{j}, v_{j}\right) \rightarrow(\eta, u, v) \quad \text { in } \quad \mathbb{R} \times \mathbb{W}_{q} \times \mathbb{W}_{q}
$$

Clearly, $u=0$ or $v=0$. Observing that

$$
\partial_{a} v_{j}-\Delta_{D} v_{j}=-\beta_{1} v_{j}^{2}+\beta_{2} u_{j} v_{j} \geqslant-\beta_{1} v_{j}^{2}, \quad v_{j}(0)=\xi V_{j},
$$

whence $v_{j} \geqslant v_{\xi}$ by Lemma A.1, we deduce $v \geqslant v_{\xi}$ and so $u=0$ since $(\eta, u, v) \in \mathfrak{C}_{2}^{\prime} \backslash \mathfrak{C}_{2}$. Therefore, $(\eta, u, v)=\left(\eta, 0, v_{\xi}\right)$ by the uniqueness statement of Theorem A.4 A similar, but simpler argument as in Lemma 3.7 (see also [20, Lem.6.5.3] or [29, Lem.4.5]) then implies that $1 / \eta$ is an eigenvalue of $H_{\left[-\alpha_{2} v_{\xi}\right]}$ with a positive eigenvector, that is, $\eta=\eta_{1}$ by Lemma A.3 and 4.1 yielding the contradiction that $(\eta, u, v)$ coincides with $\left(\eta_{1}, 0, v_{\xi}\right)$. Consequently, $\mathfrak{C}_{2}=\mathfrak{C}_{2}^{\prime}$ does not leave $\mathbb{R}^{+} \times \dot{\mathbb{W}}_{q}^{+} \times \dot{\mathbb{W}}_{q}^{+}$except at $\left(\eta_{1}, 0, v_{\xi}\right)$, and we conclude that alternative (ii) above is impossible. An argument similar to the proof of Lemma 3.7 shows that alternative (iii) can also be ruled out. Therefore, the only remaining possibility is that $\mathfrak{C}_{2}^{\prime}=\mathfrak{C}_{2}$ is unbounded in $\mathbb{R}^{+} \times \dot{\mathbb{W}}_{q}^{+} \times \dot{\mathbb{W}}_{q}^{+}$.

It remains to prove that there is no other bifurcation point on the semi-trivial branches.

Corollary 4.3. There is no other bifurcation point on $\mathfrak{B}_{2}$ or $\mathfrak{B}_{1}$ to positive coexistence solutions than $\left(\eta_{1}, 0, v_{\xi}\right) \in \mathfrak{B}_{2}$.

Proof. If $\left(\eta, 0, v_{\xi}\right) \in \mathfrak{B}_{2}$ is a bifurcation point to positive coexistence solutions, then $1=\eta r\left(H_{\left[-\alpha_{2} v_{\xi}\right]}\right)$ as in the proof of Corollary 3.8 and Lemma 3.7 by using (4.6), whence $\eta=\eta_{1}$.

Suppose that there is a bifurcation point $\left(\eta, u_{\eta}, 0\right)$ on $\mathfrak{B}_{1}$ to positive coexistence solutions. Then we deduce $1=\xi r\left(\hat{H}_{\left[-\beta_{2} u_{\eta}\right]}\right)$ as in the proof of Corollary 3.8 and Lemma 3.7 However, this is not possible since $\xi>1$ and $1=r\left(\hat{H}_{[0]}\right)<r\left(\hat{H}_{\left[-\beta_{2} u_{\eta}\right]}\right)$ by 2.4 and Lemma A.3. 
This completes the proof of Theorem 1.2. The proof of the following characterization of $\mathfrak{C}_{2}$ is the same as for Corollary 3.9 .

Corollary 4.4. The continuum $\mathfrak{C}_{2}$ is unbounded with respect to both the parameter $\eta$ and the u-component in $\mathbb{W}_{q}$, or with respect to the v-component in $\mathbb{W}_{q}$. If $(1.13)$ holds for some $s>0$, then $\mathfrak{C}_{2}$ is unbounded with respect to the u-component in $\mathbb{W}_{q}$.

\section{Competing Systems: Proof of Theorem 1.4}

We next consider (1.14)-(1.15) subject to (1.3)-(1.4). The simplest case is $\xi \leqslant 1$ when no coexistence solutions exist:

Lemma 5.1. If $\xi \leqslant 1$, then there is no solution $(\eta, u, v)$ in $\mathbb{R}^{+} \times \mathbb{W}_{q}^{+} \times \dot{\mathbb{W}}_{q}^{+}$to (1.14)-(1.15) subject to (1.3)-(1.4).

Proof. Let $(\eta, u, v) \in \mathbb{R}^{+} \times \mathbb{W}_{q}^{+} \times \dot{\mathbb{W}}_{q}^{+}$solve (1.14)-(1.15) subject to (1.3)-(1.4). Since $u \geqslant 0$, we have

$$
\partial_{a} v-\Delta_{D} v \leqslant-\beta_{1} v^{2} \text { on } J \times \Omega, \quad v(0)=\xi V,
$$

and thus $z^{\prime}(a) \leqslant-\lambda_{1} z(a)$ for $a \in J$, where

$$
z(a):=\int_{\Omega} \varphi_{1} v(a) \mathrm{d} x, \quad a \in J,
$$

and $\varphi_{1}$ is a positive eigenfunction for the principal eigenvalue $\lambda_{1}>0$ of $-\Delta_{D}$. Therefore,

$$
z(0)=\xi \int_{0}^{a_{m}} b_{2}(a) \int_{\Omega} \varphi_{1} v(a) \mathrm{d} a \mathrm{~d} x \leqslant \xi \int_{0}^{a_{m}} b_{2}(a) e^{-\lambda_{1} a} \mathrm{~d} a z(0) .
$$

Actually, this inequality is strict and $z(0)>0$ due to $v \in \dot{\mathbb{W}}_{q}^{+}$and (5.1). So $\xi>1$ by (1.6).

In the sequel, let $\xi>1$ be arbitrarily fixed. The remainder of the proof of Theorem 1.4 is then very similar to the one of Theorem 1.2, and we give merely a brief sketch of the proof mainly pointing out the differences. Due to (1.6) and Lemma A.3 we have

$$
\eta_{2}:=\eta_{2}(\xi):=\frac{1}{r\left(H_{\left[\alpha_{2} v_{\xi}\right]}\right)} \in(1, \infty) .
$$

We linearize around $\left(\eta_{2}, 0, v_{\xi}\right) \in \mathfrak{B}_{2}$ by writing solutions to (1.14)-(1.15) subject to (1.3)-(1.4) in the form $(\eta, u, v)=\left(\eta, u, v_{\xi}-w\right) \in \mathbb{R} \times \mathbb{W}_{q} \times \mathbb{W}_{q}$ with

$$
(u, w)-\hat{K}(\eta)(u, w)+\hat{R}(u, w)=0 .
$$

Hereby,

$$
\hat{K}(\eta)(u, w):=\left(\begin{array}{c}
\tilde{Z}_{1}(0, \eta U) \\
\hat{Z}_{2}\left(\beta_{2} u v_{\xi}, \xi W\right)
\end{array}\right), \quad \hat{R}(u, w):=-\left(\begin{array}{c}
\hat{Z}_{1}\left(-\alpha_{1} u^{2}+\alpha_{2} u w, 0\right) \\
\hat{Z}_{2}\left(\beta_{1} w^{2}-\beta_{2} u w, 0\right)
\end{array}\right)
$$

for $(u, w) \in \mathbb{W}_{q} \times \mathbb{W}_{q}$ with

$$
\begin{aligned}
& \hat{Z}_{1}:=\left(\partial_{a}-\Delta_{D}+\alpha_{2} v_{\xi}, \gamma_{0}\right)^{-1} \in \mathcal{L}\left(\mathbb{L}_{q} \times W_{q, D}^{2-2 / q}, \mathbb{W}_{q}\right), \\
& \hat{Z}_{2}:=\left(\partial_{a}-\Delta_{D}+2 \beta_{1} v_{\xi}, \gamma_{0}\right)^{-1} \in \mathcal{L}\left(\mathbb{L}_{q} \times W_{q, D}^{2-2 / q}, \mathbb{W}_{q}\right) .
\end{aligned}
$$

Exactly as in the previous sections we deduce with the aid of [10, 20]: there is a continuum $\mathfrak{C}_{3}^{\prime}$ of solutions $(\eta, u, v)$ to (1.14)-(1.15) subject to (1.3)-1.4) in $\mathbb{R} \times \mathbb{W}_{q} \times \mathbb{W}_{q}$ emanating from $\left(\eta_{2}, 0, v_{\xi}\right) \in \mathfrak{B}_{2}$ and satisfying the alternatives

(i) $\mathfrak{C}_{3}^{\prime}$ is unbounded in $\mathbb{R} \times \mathbb{W}_{q} \times \mathbb{W}_{q}$, or

(ii) there is $\eta \in\{\zeta \in \mathbb{R} ; \operatorname{dim}(\operatorname{ker}(1-\hat{K}(\zeta))) \geqslant 1\}$ with $\eta \neq \eta_{2}$ and $\left(\eta, 0, v_{\xi}\right) \in \mathfrak{C}_{3}^{\prime}$, or

(iii) there is $\left(\eta, u, v_{\xi}-w\right) \in \mathfrak{C}_{3}^{\prime}$ with $(u, w) \in \operatorname{rg}\left(1-\hat{K}\left(\eta_{2}\right)\right) \backslash\{(0,0)\}$. 
Close to $\left(\eta_{2}, 0, v_{\xi}\right)$, the continuum $\mathfrak{C}_{3}^{\prime}$ is a continuous curve in $\mathbb{R}^{+} \times \dot{\mathbb{W}}_{q}^{+} \times \dot{\mathbb{W}}_{q}^{+}$. We define

$$
\mathfrak{C}_{3}:=\mathfrak{C}_{3}^{\prime} \cap\left(\mathbb{R}^{+} \times \dot{\mathbb{W}}_{q}^{+} \times \dot{\mathbb{W}}_{q}^{+}\right) \neq \varnothing,
$$

and note that $\eta>1$ for $(\eta, u, v) \in \mathfrak{C}_{3}$ by reproducing the proof of Lemma 5.1 .

Lemma 5.2. The continuum $\mathfrak{C}_{3}$ is either unbounded in $\mathbb{R}^{+} \times \dot{\mathbb{W}}_{q}^{+} \times \dot{\mathbb{W}}_{q}^{+}$or joins $\mathfrak{B}_{2}$ with $\mathfrak{B}_{1}$.

Proof. Assume that $\mathfrak{C}_{3}=\mathfrak{C}_{3}^{\prime}$, that is, $\mathfrak{C}_{3}^{\prime} \subset \mathbb{R}^{+} \times \dot{\mathbb{W}}_{q}^{+} \times \dot{\mathbb{W}}_{q}^{+}$. Then alternative (ii) above is impossible while alternative (iii) can be ruled out with the same argument as in the proof of Lemma 3.7 Thus, if $\mathfrak{C}_{3}=\mathfrak{C}_{3}^{\prime}$, then it is unbounded in $\mathbb{R}^{+} \times \dot{\mathbb{W}}_{q}^{+} \times \dot{\mathbb{W}}_{q}^{+}$. Suppose that $\mathfrak{C}_{3}$ is a proper subset of $\mathfrak{C}_{3}^{\prime}$. Let $\left(\left(\eta_{j}, u_{j}, v_{j}\right)\right)_{j \in \mathbb{N}}$ be a sequence in $\mathfrak{C}_{3}$ converging toward $(\eta, u, v) \in \mathfrak{C}_{3}^{\prime} \backslash \mathfrak{C}_{3}$ with $(\eta, u, v) \neq\left(\eta_{2}, 0, v_{\xi}\right)$, so $u=0$ or $v=0$. As the only solutions close to $\mathfrak{B}_{0}$ lie on the curve $\mathfrak{B}_{1}$, the case $(u, v)=(0,0)$ is impossible. If $u=0$ but $v \neq 0$, then $(\eta, u, v)=(\eta, 0, v)$ and so, since $\xi>1, v=v_{\xi}$ by Theorem A.4 A similar, but simpler argument as in Lemma 3.7 (see also [20, Lem.6.5.3] or [29, Lem.4.5]) then implies that $\eta=\eta_{2}$. This yields the contradiction $(\eta, u, v)=\left(\eta_{2}, 0, v_{\xi}\right)$. Therefore, the only remaining possibility is that $v=0$ but $u \neq 0$ from which $(\eta, u, v)=\left(\eta, u_{\eta}, 0\right) \in \mathfrak{B}_{1}$ according to (1.14), 1.3), and Theorem A.4 This proves the claim.

The next lemma implies, in particular, that if $\mathfrak{C}_{3}$ is unbounded in $\mathbb{R}^{+} \times \dot{\mathbb{W}}_{q}^{+} \times \dot{\mathbb{W}}_{q}^{+}$, then it is unbounded with respect to the parameter $\eta$ :

Lemma 5.3. Given $M>1$ there is $c(M)>0$ such that $\|u\|_{\mathbb{W}_{q}}+\|v\|_{\mathbb{W}_{q}} \leqslant c(M)$ whenever $(\eta, u, v) \in \mathfrak{C}_{3}$ with $\eta \leqslant M$.

Proof. Let $(\eta, u, v) \in \mathfrak{C}_{3}$ with $\eta \leqslant M$. Recall $\xi, \eta>1$ and observe

$$
\partial_{a} v-\Delta_{D} v=-\beta_{1} v^{2}-\beta_{2} u v \leqslant-\beta_{1} v^{2}, \quad v(0)=\xi V,
$$

whence

$$
0 \leqslant v(a) \leqslant v_{\xi}(a) \leqslant \kappa \xi^{2}, \quad a \in J,
$$

by Lemma A.1 and Theorem A.4. Similarly,

$$
0 \leqslant u(a) \leqslant u_{\eta}(a) \leqslant \kappa \eta^{2} \leqslant \kappa M^{2}, \quad a \in J .
$$

Hence

$$
\|u(a)\|_{\infty}+\|v(a)\|_{\infty} \leqslant c(M), \quad a \in J .
$$

and we conclude with the help of Lemma 2.1

To show that $\mathfrak{C}_{3}$ joins $\mathfrak{B}_{2}$ with $\mathfrak{B}_{1}$ for certain values of $\xi$, we require the following auxiliary result. Recall that $\varphi_{1}$ is the positive eigenfunction of $-\Delta_{D}$ corresponding to the principal eigenvalue $\lambda_{1}>0$ with $\left\|\varphi_{1}\right\|_{\infty}=1$.

Lemma 5.4. Set $\mu_{1}:=\lambda_{1}+\alpha_{2} \kappa \xi^{2}$ and $m_{0}:=e^{\alpha_{2} \kappa \xi^{2} a_{m}}$. Given $\eta>m_{0}$ let $z_{\eta}(a):=f_{\eta}(a) \varphi_{1}, a \in J$, where

$$
f_{\eta}(a):=\frac{\mu_{1}}{c_{\eta} \mu_{1} e^{\mu_{1} a}-\alpha_{1}}, \quad a \in J, \quad c_{\eta}:=\frac{\alpha_{1}}{\mu_{1}} \frac{\eta-e^{-\lambda_{1} a_{m}}}{\eta-m_{0}} .
$$

Then $z_{\eta}$ is increasing in $\eta \in\left(m_{0}, \infty\right)$ and $z_{\eta} \leqslant u$ on $J \times \Omega$ for any $(\eta, u, v) \in \mathfrak{C}_{3}$ with $\eta>m_{0}$.

Proof. Note that $f_{\eta}^{\prime}+\mu_{1} f_{\eta}=-\alpha_{1} f_{\eta}^{2}$, whence

$$
\partial_{a} z_{\eta}-\Delta_{D} z_{\eta}=-\alpha_{1} z_{\eta}^{2}-\alpha_{2} \kappa \xi^{2} z_{\eta}-F \text { on } J \times \Omega
$$

where $F:=\alpha_{1}\left(f_{\eta}-z_{\eta}\right) z_{\eta} \geqslant 0$. Due to the definition of $c_{\eta}>\alpha_{1} / \mu_{1}$ and (1.6), it is easily seen that $z_{\eta}(0) \leqslant \eta Z_{\eta}$. The comparison principle stated in LemmaA.2, (5.4), and (1.14) then yield $u \geqslant z_{\eta}$ on $J \times \Omega$ for any $(\eta, u, v) \in \mathfrak{C}_{3}$ with $\eta>m_{0}$. That $z_{\eta}$ is increasing in $\eta \in\left(m_{0}, \infty\right)$ follows from $\partial_{\eta} f_{\eta}(a)>0$ for $a \in J$. 
We remark that 2.4), Lemma 5.4, Lemma A.3, and Theorem A.4 ensure that both maps $\eta \mapsto r\left(\hat{H}_{\left[\beta_{2} z_{\eta}\right]}\right)$ and $\eta \mapsto r\left(\hat{H}_{\left[\beta_{2} u_{\eta}\right]}\right)$ belong to $C\left(\left(m_{0}, \infty\right),(0,1)\right)$ and are strictly decreasing with $r\left(\hat{H}_{\left[\beta_{2} u_{\eta}\right]}\right) \leqslant r\left(\hat{H}_{\left[\beta_{2} z_{\eta}\right]}\right)$ for $\eta>m_{0}$ and $\lim _{\eta \rightarrow 0} r\left(\hat{H}_{\left[\beta_{2} u_{\eta}\right]}\right)=1$, hence

$$
\left(\lim _{\eta \rightarrow \infty} r\left(\hat{H}_{\left[\beta_{2} u_{\eta}\right]}\right)\right)^{-1} \geqslant\left(\lim _{\eta \rightarrow \infty} r\left(\hat{H}_{\left[\beta_{2} z_{\eta}\right]}\right)\right)^{-1}=: N \in(1, \infty]
$$

is well-defined.

Remark 5.5. As Lemma 5.4 ensures $\left\|z_{\eta}\right\|_{\infty} \rightarrow \infty$ for $\eta \rightarrow \infty$, we conjecture $N=\infty$.

Note that for any $\xi \in(1, N)$ there is a unique $\eta_{3}:=\eta_{3}(\xi)>1$ such that

$$
\xi r\left(\hat{H}_{\left[\beta_{2} u_{\eta_{3}}\right]}\right)=1 .
$$

In this case, the continuum $\mathfrak{C}_{3}$ connects $\mathfrak{B}_{2}$ with $\mathfrak{B}_{1}$ and the value of $\eta_{3}$ determines the point where $\mathfrak{C}_{3}$ joins up with $\mathfrak{B}_{1}$ :

Corollary 5.6. If $\xi \in(1, N)$, then $\mathfrak{C}_{3}$ joins up with $\mathfrak{B}_{1}$ at the point $\left(\eta_{3}, u_{\eta_{3}}, 0\right)$.

Proof. Let $\xi \in(1, N)$. If $(\eta, u, v) \in \mathfrak{C}_{3}$ with $\eta>m_{0}$, then $u \geqslant z_{\eta}$ by Lemma 5.4, hence

$$
1=r\left(\xi \hat{H}_{\left[\beta_{1} v+\beta_{2} u\right]}\right) \leqslant r\left(\xi \hat{H}_{\left[\beta_{2} z_{\eta}\right]}\right)
$$

owing to Lemma A.3 and (2.8). Since the right hand side tends to $\xi / N<1$ as $\eta \rightarrow \infty$, there must be some $M=M(\xi)>1$ such that $\eta \leqslant M$ for any $(\eta, u, v) \in \mathfrak{C}_{3}$. Thus $\mathfrak{C}_{3}$ joins up with $\mathfrak{B}_{1}$ due to Lemma 5.2 and Lemma 5.3. say, at $\left(\hat{\eta}, u_{\hat{\eta}}, 0\right)$. To determine $\hat{\eta}$ we first recall that

$$
(\eta, u, v)=\left(\eta, u_{\eta}-w, v\right) \in \mathbb{R} \times \mathbb{W}_{q} \times \mathbb{W}_{q}
$$

solves (1.14)-(1.15) subject to (1.3)-(1.4) if and only if $(\eta, w, v) \in \mathbb{R} \times \mathbb{W}_{q} \times \mathbb{W}_{q}$ solves

$$
\begin{array}{lr}
\partial_{a} w-\Delta_{D} w=\alpha_{1} w^{2}-2 \alpha_{1} u_{\eta} w+\alpha_{2} u_{\eta} v-\alpha_{2} v w, & w(0)=\eta W, \\
\partial_{a} v-\Delta_{D} v=-\beta_{1} v^{2}-\beta_{2} v\left(u_{\eta}-w\right), & v(0)=\xi V,
\end{array}
$$

where we put

$$
W:=\int_{0}^{a_{m}} b_{1}(a) w(a) \mathrm{d} a, \quad V:=\int_{0}^{a_{m}} b_{2}(a) v(a) \mathrm{d} a
$$

Introducing

$$
T:=\left(\partial_{a}-\Delta_{D}, \gamma_{0}\right)^{-1} \in \mathcal{L}\left(\mathbb{L}_{q} \times W_{q, D}^{2-2 / q}, \mathbb{W}_{q}\right)
$$

and the operators

$$
K_{*}(\eta)(w, v):=\left(\begin{array}{c}
T\left(-2 \alpha_{1} u_{\eta} w+\alpha_{2} u_{\eta} v, \eta W\right) \\
T\left(-\beta_{1} u_{\eta} v, \xi V\right)
\end{array}\right), \quad R_{*}(w, v):=-\left(\begin{array}{c}
T\left(\alpha_{1} w^{2}-\alpha_{2} v w, 0\right) \\
T\left(-\beta_{1} v^{2}+\beta_{2} w v, 0\right)
\end{array}\right)
$$

acting on $(w, v) \in \mathbb{W}_{q} \times \mathbb{W}_{q}$, equations (5.7), (5.8) are equivalent to

$$
(w, v)-K_{*}(\eta)(w, v)+R_{*}(w, v)=0 .
$$

The operators $K_{*}$ and $R_{*}$ possess the properties stated in (3.14)-(3.16). Now, as $\mathfrak{C}_{3}$ joins up with $\mathfrak{B}_{1}$ at $\left(\hat{\eta}, u_{\hat{\eta}}, 0\right)$, there is a sequence $\left(\left(\eta_{j}, u_{j}, v_{j}\right)\right)_{j}$ in $\mathfrak{C}_{3}$ converging to $\left(\hat{\eta}, u_{\hat{\eta}}, 0\right)$. Set $w_{j}:=u_{\eta_{j}}-u_{j}$ and note that $w_{j} \in \mathbb{W}_{q}^{+}$according to (5.5). As $u_{\eta}$ depends continuously on $\eta$, formulation (5.9) and the properties of $K_{*}$ and $R_{*}$ readily imply (see, e.g., the proof of [20, Lem.6.5.3] or Lemma 3.7] that

$$
\frac{\left(w_{j}, v_{j}\right)}{\left\|\left(w_{j}, v_{j}\right)\right\|_{\mathbb{W}_{q} \times \mathbb{W}_{q}}}
$$


converges to some eigenvector $(\phi, \psi) \in \mathbb{W}_{q}^{+} \times \mathbb{W}_{q}^{+}$of $K_{*}(\hat{\eta})$ associated to the eigenvalue 1 and thus satisfying (5.7), (5.8) with $\eta=\hat{\eta}$ when higher order terms are neglected:

$$
\begin{aligned}
\partial_{a} \phi-\Delta_{D} \phi & =-2 \alpha_{1} u_{\hat{\eta}} \phi+\alpha_{2} u_{\hat{\eta}} \psi, & & \phi(0)=\hat{\eta} \Phi, \\
\partial_{a} \psi-\Delta_{D} \psi & =-\beta_{2} u_{\hat{\eta}} \psi, & & \psi(0)=\xi \Psi .
\end{aligned}
$$

Suppose $\psi=0$. Then the first equation yields $\phi(0)=\hat{\eta} H_{\left[2 \alpha_{1} u_{\hat{\eta}}\right]} \phi(0)$ and thus, since $\phi(0) \in W_{q, D}^{2-2 / q} \backslash\{0\}$, we obtain from Lemma A.3 and (2.9) the contradiction

$$
1 \leqslant \hat{\eta} r\left(H_{\left[2 \alpha_{1} u_{\hat{\eta}}\right]}\right)<\hat{\eta} r\left(H_{\left[\alpha_{1} u_{\hat{\eta}}\right]}\right)=1 .
$$

Therefore, $\psi \neq 0$ and hence $\psi(0) \in W_{q, D}^{2-2 / q} \backslash\{0\}$. The equation for $\psi$ ensures $\psi(0)=\xi \hat{H}_{\left[\beta_{2} u_{\hat{\eta}}\right]} \psi(0)$, whence $\xi r\left(\hat{H}_{\left[\beta_{2} u_{\hat{\eta}}\right]}\right)=1$. We conclude $\hat{\eta}=\eta_{3}$ according to [5.6).

Finally, we show that $\mathfrak{C}_{3}$ connects the two semi-trivial branches if assumption (1.16) holds.

Corollary 5.7. Suppose $(1.16)$ and let $\xi>1$ be arbitrary. Then the $\eta$-projection of $\mathfrak{C}_{3}$ is contained in the interval $(1, \xi]$. In particular, $\mathfrak{C}_{3}$ joins $\mathfrak{B}_{2}$ with $\mathfrak{B}_{1}$.

Proof. Given $\Phi \in W_{q, D}^{2-2 / q,+}$ and $u, v \in \mathbb{W}_{q}^{+}$we have

$$
\begin{aligned}
\left(H_{\left[\alpha_{1} u+\alpha_{2} v\right]}-\hat{H}_{\left[\beta_{1} v+\beta_{2} u\right]}\right) \Phi= & \int_{0}^{a_{m}}\left(b_{1}(a)-b_{2}(a)\right) \Pi_{\left[\beta_{1} v+\beta_{2} u\right]}(a, 0) \Phi \mathrm{d} a \\
& +\int_{0}^{a_{m}} b_{1}(a)\left(\Pi_{\left[\alpha_{1} u+\alpha_{2} v\right]}(a, 0)-\Pi_{\left[\beta_{1} v+\beta_{2} u\right]}(a, 0)\right) \Phi \mathrm{d} a .
\end{aligned}
$$

Since $\alpha_{1} u+\alpha_{2} v \leqslant \beta_{1} v+\beta_{2} u$ by (1.16), the parabolic maximum principle implies

$$
\left(\Pi_{\left[\alpha_{1} u+\alpha_{2} v\right]}(a, 0)-\Pi_{\left[\beta_{1} v+\beta_{2} u\right]}(a, 0)\right) \Phi \geqslant 0 \text { on } \Omega, \quad a \in J,
$$

whence $H_{\left[\alpha_{1} u+\alpha_{2} v\right]} \geqslant \hat{H}_{\left[\beta_{1} v+\beta_{2} u\right]}$ by the above equality from which

$$
r\left(H_{\left[\alpha_{1} u+\alpha_{2} v\right]}\right) \geqslant r\left(\hat{H}_{\left[\beta_{1} v+\beta_{2} u\right]}\right)
$$

due to [1, Thm.3.2(v)]. Thus, given $(\eta, u, v) \in \mathfrak{C}_{3}$ we have

$$
1=\xi r\left(\hat{H}_{\left[\beta_{1} v+\beta_{2} u\right]}\right) \leqslant \xi r\left(H_{\left[\alpha_{1} u+\alpha_{2} v\right]}\right)=\frac{\xi}{\eta}
$$

by (2.8) and (2.7). So the $\eta$-projection of $\mathfrak{C}_{3}$ is contained in $(1, \xi]$. Due to Lemma 5.2 and Lemma 5.3, this in particular implies that $\mathfrak{C}_{3}$ joins $\mathfrak{B}_{2}$ with $\mathfrak{B}_{1}$.

This completes the proof of Theorem 1.4

\section{Appendix A. Auxiliary Results: Semi-Trivial Branches}

In this appendix we collect certain results regarding the parameter-dependent equation

$$
\partial_{a} u-\Delta_{D} u=-\alpha_{1} u^{2}, \quad u(0, \cdot)=\eta \int_{0}^{a_{m}} b_{1}(a) u(a, \cdot) \mathrm{d} a .
$$

Most of these results have been proved in [29].

Suppose (1.5) and (1.6) in the following. We first recall a comparison principle (see [29, Lem.3.2]) for parabolic equations with nonlocal initial conditions of the form A.1 which, in particular, guarantees uniqueness of positive solutions: 
Lemma A.1. Let $\eta>1$ and $F \in \mathbb{L}_{q}^{+}$. Suppose $u, v \in \dot{\mathbb{W}}_{q}^{+}$satisfy either

$$
\begin{array}{ll}
\partial_{a} u-\Delta_{D} u=-\alpha_{1} u^{2}+F \quad \text { in } J \times \Omega, & u(0) \geqslant \eta \int_{0}^{a_{m}} b_{1}(a) u(a) \mathrm{d} a, \\
\partial_{a} v-\Delta_{D} v=-\alpha_{1} v^{2} \text { in } J \times \Omega, & v(0)=\eta \int_{0}^{a_{m}} b_{1}(a) v(a) \mathrm{d} a,
\end{array}
$$

or

$$
\begin{array}{ll}
\partial_{a} u-\Delta_{D} u=-\alpha_{1} u^{2} \quad \text { in } J \times \Omega, & u(0)=\eta \int_{0}^{a_{m}} b_{1}(a) u(a) \mathrm{d} a, \\
\partial_{a} v-\Delta_{D} v=-\alpha_{1} v^{2}-F \quad \text { in } J \times \Omega, & v(0) \leqslant \eta \int_{0}^{a_{m}} b_{1}(a) v(a) \mathrm{d} a .
\end{array}
$$

Then $u \geqslant v$.

Along the lines of the proof of [29, Lem.3.2] one may also derive the following variant:

Lemma A.2. Let $\eta>1$ and $F \in \mathbb{L}_{q}^{+}$. Let $R>0$ and suppose $u, w, v \in \dot{\mathbb{V}}_{q}^{+}$with $v \leqslant R$ on $J \times \Omega$ satisfy

$$
\begin{array}{ll}
\partial_{a} u-\Delta_{D} u=-\alpha_{1} u^{2}-\alpha_{2} u v \quad \text { in } J \times \Omega, & u(0)=\eta \int_{0}^{a_{m}} b_{1}(a) u(a) \mathrm{d} a, \\
\partial_{a} w-\Delta_{D} w=-\alpha_{1} w^{2}-\alpha_{2} R w-F \quad \text { in } J \times \Omega, & w(0) \leqslant \eta \int_{0}^{a_{m}} b_{1}(a) w(a) \mathrm{d} a .
\end{array}
$$

Then $u \geqslant w$.

Properties of solutions to A.1] are connected to operators of the form $H_{[h]}$ as introduced in Section 2 . The next lemma is a consequence of the famous Krein-Rutman theorem [1, Thm.3.2] and gives information about the spectral radii of such operators. We refer to [29. Lem.3.1] for a proof. Actually, the proof of Lemma A.1 given in [29] is based on the next lemma.

Lemma A.3. For $h \in C^{\varrho}(J, C(\bar{\Omega}))$ with $\varrho>0$, the operator $H_{[h]} \in \mathcal{K}\left(W_{q, D}^{2-2 / q}\right)$ is strongly positive, i.e. (2.3) holds. In particular, the spectral radius $r\left(H_{[h]}\right)>0$ is a simple eigenvalue with an eigenfunction $B_{[h]}$ belonging to int $\left(W_{q, D}^{2-2 / q,+}\right)$. It is the only eigenvalue of $H_{[h]}$ with a positive eigenfunction. Moreover, if $h$ and $g$ both belong to $C^{\varrho}(J, C(\bar{\Omega}))$ with $g \geqslant h$ but $g \not \equiv$, then $r\left(H_{[g]}\right)<r\left(H_{[h]}\right)$.

Finally, we gather results from [29] about properties of solutions to (A.1) being fundamental for the investigation of (1.1)-(1.4). Recall that $\lambda_{1}>0$ is the principal eigenvalue of $-\Delta_{D}$ with positive eigenfunction $\varphi_{1}$ (normalized such that $\left\|\varphi_{1}\right\|_{\infty}=1$ ).

Theorem A.4. For each $\eta>1$ there is a unique solution $u_{\eta} \in \mathbb{W}_{q}^{+} \backslash\{0\}$ to equation A.1. The mapping $\left(\eta \mapsto u_{\eta}\right) \in C^{\infty}\left((1, \infty), \mathbb{W}_{q}\right)$ is real analytic with $\left\|u_{\eta}\right\| \mathbb{W}_{q} \rightarrow 0$ as $\eta \rightarrow 1$ and $\left\|u_{\eta}\right\| \mathbb{W}_{q} \rightarrow \infty$ as $\eta \rightarrow \infty$ There is $\kappa>0$ such that, for $\eta>1$,

$$
\kappa \eta^{2} \geqslant u_{\eta}(a) \geqslant \frac{\lambda_{1}}{\alpha_{1}} \frac{\eta-1}{\eta\left(e^{\lambda_{1} a}-1\right)+1-e^{-\lambda_{1}\left(a_{m}-a\right)}} \varphi_{1} \quad \text { on } \Omega, \quad a \in J,
$$

and $\frac{\partial}{\partial \eta} u_{\eta}(a) \in \operatorname{int}\left(W_{q, D}^{2-2 / q,+}\right)$ for $a \in J$. If $\eta_{1}>\eta_{2}$, then $u_{\eta_{1}} \geqslant u_{\eta_{2}}$. Finally, if $\eta \leqslant 1$, then (A.1) has no solution in $\mathbb{W}_{q}^{+} \backslash\{0\}$.

The proof of this theorem is given in [29, Thm.2.1, Cor.3.3, Lem.3.6, Lem.3.7] except for the analyticity of the mapping $\eta \mapsto u_{\eta}$. However, this follows exactly as in the proof of [29, Thm.2.1] (see subsection 3.3 therein) by taking into account the real analyticity of the mapping

$$
\Gamma:(1, \infty) \times \mathbb{W}_{q} \rightarrow \mathbb{L}_{q} \times W_{q, D}^{2-2 / q}, \quad(\eta, u) \mapsto\left(\partial_{a} u-\Delta_{D} u+\alpha_{1} u^{2}, u(0)-\eta \int_{0}^{a_{m}} b_{1}(a) u(a, \cdot) \mathrm{d} a\right)
$$

and invoking the implicit function theorem for analytic maps, e.g. [6, Thm.4.5.4]. 


\section{REFERENCES}

[1] H. Amann. Fixed point equations and nonlinear eigenvalue problems in ordered Banach spaces. SIAM Rev. 18 (1976), no. 4, 620-709.

[2] H. Amann. Linear and Quasilinear Parabolic Problems, Volume I: Abstract Linear Theory. Birkhäuser 1995.

[3] H. Amann. Compact embeddings of vector-valued Sobolev and Besov spaces. Glas. Mat. Ser. III 3555 (2000), no. 1, 161-177.

[4] J. Blat, K.J. Brown. Bifurcation of steady-state solutions in predator-prey and competition systems. Proc. Royal Soc. Edinburgh 97A (1984), 21-34.

[5] J. Blat, K.J. Brown. Global bifurcation of positive solutions in some systems of elliptic equations. SIAM J. Math. Anal. 17 (1986), 1339-1353.

[6] B. Buffoni, J. Toland. Analytic Theory of Global Bifurcation. Princeton Series in Applied Mathematics, Princeton University Press, 2003.

[7] R.S. Cantrell, C. Cosner. On the steady-state problem for the Volterra-Lotka competition model with diffusion. Houston J. Math. 13 (1987), 337-352.

[8] A. Casal, J.C. Eilbeck, J. J. López-Gómez. Existence and uniqueness of coexistence states for a predator-prey model with diffusion. Differential Integral Equations 7 (1994), 411-439.

[9] C. Cosner, A.C. Lazer. Stable coexistence states in the Volterra-Lotka competition model with diffusion. SIAM J. Appl. Math. 44 (1984), 1112-1132.

[10] M.C. Crandall, P.H. Rabinowitz. Bifurcation from simple eigenvalues. J. Functional Analysis 8 (1971), 321-340.

[11] E.N. Dancer. On positive solutions of some pairs of differential equations. Trans. Amer. Math. Soc. 284 (1984), 729-743.

[12] E.N. Dancer. On positive solutions of some pairs of differential equations. II. J. Differential Equations 60 (1985), 236-258.

[13] E.N. Dancer, J. López-Gómez, R. Ortega. On the spectrum of some linear noncooperative elliptic systems with radial symmetry. Differential Integral Equations 8 (1995), 515-523.

[14] D. Daners, P. Koch-Medina. Abstract Evolution Equations, Periodic Problems, and Applications. Pitman Res. Notes Math. Ser., 279, Longman, Harlow 1992.

[15] M. Delgado, M. Molina-Becerra, A. Suárez. A nonlinear age-dependent model with spatial diffusion. J. Math. Anal. Appl. 313 (2006), 366-380.

[16] M. Delgado, M. Molina-Becerra, A. Suárez. Nonlinear age-dependent diffusive equations: A bifurcation approach. J. Differential Equations 244 (2008), 2133-2155.

[17] T. Kato. Perturbation Theory for Linear Operators. Second Edition. Grundlehren der mathematischen Wissenschaften 132, Springer 1976.

[18] A. Leung. Monotone schemes for semilinear elliptic systems related to ecology. Math. Methods Appl. Sci. 4 (1982), 272-285.

[19] J. López-Gómez, R. Pardo. On the uniqueness and stability of coexistence states for some predator-prey models with diffusion. Proceedings of Dynamic Systems and Applications, Vol. 1 (Atlanta, GA, 1993), 221-228, Dynamic, Atlanta, GA, 1994.

[20] J. López-Gómez. Spectral Theory and Nonlinear Functional Analysis. Research Notes in Mathematics. Chapman \& Hall 2001.

[21] J. López-Gómez, M. Molina-Meyer. Superlinear indefinite systems: beyond Lotka-Volterra models. J. Differential Equations 221 (2006), 343-411.

[22] J. López-Gómez. The steady-states of a non-cooperative model of nuclear reactors. J. Differential Equations 246 (2009), 358372.

[23] P.H. Rabinowitz. Some global results for nonlinear eigenvalue problems. J. Functional Analysis 7 (1971), 487-513.

[24] A. Schiaffino, A. Tesei. Competition systems with Dirichlet boundary conditions. J. Math. Biol. 15 (1982), 93-105.

[25] Ch. Walker. Positive equilibrium solutions for age and spatially structured population models. SIAM J. Math. Anal. 41 (2009), 1366-1387.

[26] Ch. Walker. Global bifurcation of positive equilibria in nonlinear population models. J. Differential Equations 248 (2010), 17561776.

[27] Ch. Walker. Coexistence steady-states in a predator-prey model. Arch. Math. 95 (2010), 87-99.

[28] Ch. Walker. Bifurcation of positive equilibria in nonlinear structured population models with varying mortality rates. To appear in: Ann. Mat. Pura Appl. (arXiv:1002.1788 math.AP])

[29] Ch. Walker. On positive solutions of some system of reaction-diffusion equations with nonlocal initial conditions. To appear in: J. Reine Angew. Math. (arXiv:1003.4698 math.AP]).

[30] G.F. Webb. Population models structured by age, size, and spatial position. In: P. Magal, S. Ruan (eds.) Structured population models in biology and epidemiology. Lecture Notes in Mathematics, Vol. 1936. Springer, Berlin, 2008.

[31] L. Zhou, C.V. Pao. Asymptotic behavior of a competition-diffusion system in population dynamics. Nonlinear Anal. 6 (1982), 1163-1184.

Leibniz Universität Hannover, Institut fÜr Angewandte Mathematik, Welfengarten 1, D-30167 HanNOVER, GERMANY

E-mail address: walker@ifam.uni-hannover.de 